\title{
A Piecewise-Diffusion Model of New-Product Demands
}

\author{
Shun-Chen Niu \\ School of Management, University of Texas at Dallas, P.O. Box 830688, \\ Richardson, Texas 75083-0688, scniu@utdallas.edu
}

\begin{abstract}
The Bass Model (BM) is a widely-used framework in marketing for the study of new-product sales growth. Its usefulness as a demand model has also been recognized in production, inventory, and capacity-planning settings. The BM postulates that the cumulative number of adopters of a new product in a large population approximately follows a deterministic trajectory whose growth rate is governed by two parameters that capture (i) an individual consumer's intrinsic interest in the product, and (ii) a positive force of influence on other consumers from existing adopters. A finite-population purebirth-process (re)formulation of the BM, called the Stochastic Bass Model (SBM), was proposed recently by the author in a previous paper, and it was shown that if the size of the population in the SBM is taken to infinity, then the SBM and the BM agree (in probability) in the limit. Thus, the SBM "expands" the BM in the sense that for any given population size, it is a well-defined model. In this paper, we exploit this expansion and introduce a further extension of the SBM in which demands of a product in successive time periods are governed by a history-dependent family of SBMs (one for each period) with different population sizes. A sampling theory for this extension, which we call the Piecewise-Diffusion Model (PDM), is also developed. We then apply the theory to a typical product example, demonstrating that the PDM is a remarkably accurate and versatile framework that allows us to better understand the underlying dynamics of new-product demands over time. Joint movement of price and advertising levels, in particular, is shown to have a significant influence on whether or not consumers are "ready" to participate in product purchase.
\end{abstract}

Subject classifications: marketing: new products, buyer behavior, pricing; probability: diffusion; inventory/production: stochastic, nonstationary demand.

Area of review: Manufacturing, Service, and Supply Chain Operations.

History: Received August 2003; revision received September 2004; accepted July 2005.

\section{Introduction and Summary}

Operational decisions of firms often depend critically on demand information; indeed, demand can be taken as what drives entire supply chains. Conversely, production and marketing strategies also directly impact on demand growth. The appropriate modeling of demand is therefore of importance. This is particularly true for new products, because demands for such products have a highly dynamic and hence less predictable growth behavior. The Bass Model (BM), introduced over three decades ago (Bass 1969), is a new-product sales-growth model that has been widely used in marketing (see, e.g., Mahajan and Muller 1979, Mahajan and Wind 1986a, Mahajan et al. 1990, Parker 1994, Rogers 1995, and Mahajan et al. 2000). The model was originally developed and tested for consumer durables, but it has been found to be applicable to many other product categories as well. Furthermore, it has been generally recognized that the BM, in addition to its usefulness in marketing, can serve well as an integrated component in formulating production, inventory, and capacity-planning models (see, e.g., Cohen et al. 2000, p. 256, Table 10.1). The formulation of the BM is, however, deterministic. Consequently, it does not offer the often sought and possibly critical information on the variability of the sales trajectory of a new product. This is one of the primary motivations that prompted the initiation of work on a stochastic generalization of the BM in Niu (2002). This paper is a continuation of Niu (2002); it is concerned with the formulation, sampling-theory development, and empirical examination of a suite of stochastic new-product demand models that are further extensions of the BM.

To properly motivate the model development, we begin with a description of the BM. For $t \geqslant 0$, let $N(t)$ be the cumulative number of adopters of a product by time $t$ in a large target population; then, it is postulated that the trajectory of $N(t)$ is deterministic and that $N(t)$ can be approximated by the solution of the differential equation

$\frac{d}{d t} N(t)=[m-N(t)]\left[p+\frac{q}{m} N(t)\right]$

where $N(0) \equiv 0, m$ is the size of the population, and $p$ and $q$ are two parameters that are called, respectively, the coefficient of innovation and the coefficient of imitation. That is, $N(t)$ is assumed to grow at a rate that equals the product of $m-N(t)$, the number of individuals who have 
not yet adopted the product by time $t$, and $p+(q / m) N(t)$, a linear function in the number of existing adopters. The parameter $p$ can be interpreted as reflecting the extent of a consumer's intrinsic propensity to purchase the product, and the parameter $q$ the extent of a positive influence from an existing adopter on the entire population.

The BM can also be formulated in terms of the cumulative fractions of individuals who have adopted the product over time. For $t \geqslant 0$, let

$F(t) \equiv \frac{N(t)}{m}$;

then, Equation (1) is equivalent to

$\frac{d}{d t} F(t)=[1-F(t)][p+q F(t)]$.

Note that it is implicit in (2) that $m$ is essentially "at infinity," so that the ratio $N(t) / m$ is independent of $m$. It follows that the fraction in (2) can intuitively be thought of as the "probability" for a randomly-selected individual in an infinite population to have adopted the product by time $t$. Equation (3), together with this adoption-time-distribution interpretation for (2), is in fact often taken as the primitive definition of the BM.

While the S-shaped solution of (3) (Bass 1969, p. 218),

$F(t)=\frac{1-e^{-(p+q) t}}{1+(q / p) e^{-(p+q) t}}$,

has often been found to fit well with observed sales data, the question of whether or not there exists a suitable version of the BM that incorporates randomness into the model has been raised by many researchers (see, e.g., Jeuland 1979, Mahajan and Muller 1979, Mahajan and Peterson 1985, Eliashberg and Chatterjee 1986, Böker 1987, and Skiadas and Giovanis 1997). Stochastic formulations are desirable because they could allow greater realism in terms of microlevel interactions between consumers; they allow the sales trajectory to be dynamic, or history dependent (note that (4) does not have a dynamic dependence on the sales history; see, e.g., Trajtenberg and Yitzhaki 1989, p. 36, paragraph after Equation (4) in particular); and, most importantly, they could lead to sampling theories that are needed to support parameter estimation and to conduct interval sales forecasts (see, e.g., Schmittlein and Mahajan 1982, p. 74, top two paragraphs; Eliashberg and Chatterjee 1986, pp. 192-194; and Böker 1987, pp. 64-65).

Building on a very early model of Taga and Isii (1959), Niu (2002) recently proposed a stochastic version of the $\mathrm{BM}$, called the Stochastic Bass Model (SBM). The idea behind the formulation of the SBM is simply to replace the deterministic trajectory $N(t)$ defined in (1) by a statedependent pure birth process (see $\$ 2.1$ for a formal definition). Paralleling the BM, the birth rates in the SBM are assumed to depend on two parameters that are conceptual equivalents of $p$ and $q$. It is shown in Niu (2002) that for sufficiently large $m$, the fraction of individuals who have adopted a product by time $t$ in the SBM is, with probabil- ity close to 1 , located within any given small neighborhood of the corresponding fraction (2) in a BM with the same pair of parameter values. In other words, the SBM has been shown to be consistent with the BM in the limit.

There is, however, an important difference between the SBM and the BM, namely, that the former provides a well-defined model instance for every finite $m$, whereas the latter, defined in (2) and (3) with $m$ intended to be at infinity, does not have sufficient structure to lend itself to detailed modeling when $m$ is finite (although it does work well as an approximation). Thus, the SBM properly "expands" the BM by covering the full spectrum of population sizes. The expansion naturally suggests the possibility of using the SBMs as building blocks in the rigorous construction of new-product demand models that allow varying population sizes over time. Because the active sales-growth periods of most successful products span more than a decade, the proper development of such models has long been recognized as a challenging problem in the literature (see, e.g., Mahajan and Wind 1986b, pp. 15-16).

In this paper, we exploit the finite-population expansion in the SBM formulation and introduce a PiecewiseDiffusion Model (PDM) in which sales of a product in different time periods (years, typically) are assumed to be governed by stochastic outcomes of successive runs of a history-dependent family of SBMs. More specifically, the SBMs in the PDM are constructed to have appropriatelylinked parameters and to have differently-sized populations of "ready" (or "genuine") potential buyers of a product, both of which are explicitly tied to the cumulative sales history. The PDM is thus, in contrast with the BM, a fullydynamic model.

The PDM is in fact a versatile umbrella that covers a host of versions that progressively incorporate more marketingmix variables, i.e., price, advertising, promotion, distribution, product quality, and other information (see, e.g., Kalish and Sen 1986, pp. 90-91). At the base level, we assume that in every period, the number of ready potential adopters is a constant fraction, called the participation fraction, of those in the entire target population who have not yet adopted the product. The constant-fraction assumption is intended to approximately model the aggregate impact of all underlying marketing-mix variables. This results in a basic version of the PDM that can be useful in scenarios where detailed marketing-mix information is not available. Following the well-established spirit of a stream of prior research (see, e.g., Robinson and Lakhani 1975; Bass 1980; Horsky and Simon 1983; Kalish 1985; Kalish and Sen 1986; Jain and Rao 1990; Bass et al. 1994, 2000), we also develop versions of the PDM that explicitly account for the marginal impact of two most important marketing-mix variables, namely, price and advertising. Specifically, in the context of the PDM, we assume that the effects of price and advertising can be captured through a "response function" that for every given time period, takes as input the current price and the current advertising spending and returns as 
output a prescribed participation fraction for that period; in other words, we assume that price and advertising jointly modulate successive participation fractions. This results in a couple of more sophisticated versions of the PDM, with a set of parameters that reflect current-period effects of price and advertising. Finally, we assume that advertising also has a cumulative effect and formulate into the PDM a parameter that explicitly reflects the extent of such impact.

A rigorous sampling theory for the PDM is also developed in this paper. The theory is built on the solution of a stochastic differential equation that, when $m$ is sufficiently large, accurately describes the movement of the deviation (properly scaled) between the actual adoption trajectory and the expected adoption trajectory in the SBM. This results in a central limit theorem, on the basis of which an explicit likelihood function for successive sales in the PDM is derived. The likelihood function is then used to conduct a careful empirical validation of the PDM.

The PDM has been tested against and found to be solidly supported by data for a variety of products. In this paper, we will discuss empirical results only for a single product, namely, room air conditioners. (A thorough empirical study for seven other products will be reported in a companion paper; see Niu 2006.) This particular product is chosen because its sales trajectory has a very generic pattern, and because it has been studied, and thus benchmarked, extensively in previous literature. For this product, we also compare the empirical performance of the PDM with those of the BM, which does not explicitly include price and advertising, and the Generalized Bass Model (GBM; see Bass et al. 1994 and Krishnan et al. 1999), which is a well-established extension of the BM that includes price and advertising (the majority of other extensions of the BM includes either price or advertising, but not jointly). The PDM is found to deliver superior performance, in terms of both model fit and step-ahead forecasts. The accuracy of multiperiod step-ahead forecasts (up to five steps in a single set of forecasts without parameter update) of the PDM over that of the GBM is particularly noteworthy, with marginal reductions in mean squared errors that are in the neighborhood of $99 \%$.

The PDM is highly microscopic. Beyond fit accuracy, the PDM yields a substantial amount of surgical information that allows us to better understand the dynamics of a host of underlying characteristics that drive the demand trajectory. Some of the conclusions from our empirical analysis are highlighted next.

It is well known that parameter estimates of the BM, most critically that for $m$, are not stable (see, e.g., Srinivasan and Mason 1986, p. 177, Table 6, the " $m$ " column in particular; and Putsis and Srinivasan 2000, pp. 268-269). This strongly suggests that the BM, despite its acknowledged fit performance, is not well specified. In the PDM, $m$ is considered generic and static, typically set according to the number of households; its traditional parameter status (Bass 1969) in the BM is shifted to the participation fractions. This results in remarkably stable estimates both for the model parameters and for successive levels of cumulative market penetration, the latter of which is of particular managerial interest.

For a product that has existed for a sufficiently long duration prior to the first period of an empirical study, a consumer's intrinsic/initial interest in the product ( $p$ in the BM) is found to be (typically) of negligible relevance. We establish instead that influence from the preexisting adopters at the onset of the first period plays a dominant role. Beyond the first period, further contributions to this influence by subsequent adopters are also explicitly modeled, and accounted for, in the PDM. This dynamic feature fully formalizes the core intent of the original Bass formulation, that during the active sales-growth periods of a product, the accumulation of existing adopters should play a primary role in driving product acceptance, and hence sales. Indeed, the resulting "carry-through" (see, e.g., Bass et al. 2000, p. 102) effect on sales is an important contributing reason behind the remarkable accuracy of the PDM, especially when explicit marketing-mix influences are introduced.

As a function of time, the variance of the sales trajectory in the PDM is shown to have a bell shape. This is established via a closed-form formula (see \$2.1, Equations (13), (20), and (18)) that accurately approximates (for large $m$ ) the trajectory of the variance of the cumulative number of adoptions in the SBM. In light of the extremely complicated correlation structure of the adoption process, the identification of such a formula is significant. Apart from playing an integral role in parameter estimation, the formula is, in particular, useful in product-planning scenarios (see, e.g., Cohen et al. 2000, Ho et al. 2002, Kumar and Swaminathan 2003, and Kurawarwala and Matsuo 1996) where uncertainty in sales is of managerial concern. Indeed, we also establish empirically that variability in the sales of a product rises at a rapid pace during the early periods of active demand growth. This underscores the practical importance of quantifying variability in sales.

Joint movement of price and advertising levels is established as having a major influence on the readiness of potential buyers of a product. We show that if price fluctuations cover a sufficiently-wide range, then as prices move lower from the generally-higher initial level, price sensitivity of consumers will (typically) experience an initial climb, a levelling phase, and then a final declining phase. We also establish explicit empirical support for the intuitive beliefs that advertising lowers price sensitivity of consumers and that as prices decline, the effectiveness of advertising should increase.

The remainder of this paper is organized as follows. In $\S 2$, we review the SBM formulation in Niu (2002) and state several basic asymptotic results, including in particular a functional central limit theorem for SBMs indexed by the population size $m$; the proofs are given in the online companion to this paper available at http://or.pubs.informs. org/Pages/collect.html. A piecewise version of the SBM, 
referred to as the PSBM, is also developed in $\$ 2$. In $\S 3$, we introduce the PDM, which, in essence, is an empirical operationalization of the PSBM that incorporates both exogenous random errors and the functional central limit theorem for the SBM. In $\S 4$, we formulate particular versions of the PDM that reflect the impacts of marketingmix variables. Empirical results are presented and discussed in $§ 5$. Finally, §6 contains some concluding remarks.

\section{The SBM and a Piecewise Extension}

\subsection{The Stochastic Bass Model}

A continuous-time stochastic process with state space $\{0,1, \ldots\}$ is called a pure birth process if (i) a transition (i.e., a state change) from state $j, j \geqslant 0$, can only result in an increment to state $j+1$, and (ii) the time until such a transition occurs, given the entire history, is exponentially distributed with a rate (reciprocal of mean) that depends only on the current state (see, e.g., Ross 2003, pp. 352 and 362). For $t \geqslant 0$, let $A_{m}(t)$ be the cumulative number of adopters of a product by time $t$, where the subscript $m$ explicitly denotes the size of the target population. Suppose that $A_{m}(0)=0$; then, the stochastic formulation of the BM in Niu (2002) postulates that $\left\{A_{m}(t), t \geqslant 0\right\}$ is a pure birth process in which state $m$ is absorbing and, for $0 \leqslant j \leqslant m-1$, the transition rate from state $j$ to state $j+1$ is given by

$\lambda_{m j} \equiv(m-j)\left(\alpha+\frac{\beta}{m-1} j\right)$

where $\alpha$ and $\beta$ are two nonnegative parameters that are called, respectively, the intrinsic adoption rate and the induction rate. We will refer to $\left\{A_{m}(t), t \geqslant 0\right\}$ as an SBM with specification $(m ; \alpha, \beta)$.

The explicit individual-level interpretation of (5), which parallels in spirit Equation (1) in the BM, is as follows. The term $m-j$ is the size of the remaining potential adopters at a given time epoch. The term $\alpha+(\beta /(m-1)) j$ is the instantaneous rate for any one of these remaining individuals to adopt the product. The rate $\alpha$ formalizes the extent of an individual's inertia to purchase, independent of previous adopters; and it can be viewed as a reflection of the intrinsic appeal of the product. The rate $\beta$ formalizes the force of the total influence any one of the $j$ existing adopters has on the entire target population. The ratio $\beta /(m-1)$ formalizes the assumption that every other member of the population has an equal probability of having a "contact" with and hence being influenced by an existing adopter. Finally, if an individual has already adopted the product, all exerted influence from other existing adopters is ignored.

Observe that for every $m$, (5) defines an SBM; and by varying $m$, we have a family of SBMs indexed by $m$. Define

$B_{m}(t) \equiv \frac{A_{m}(t)}{m}$ then, it is shown in Niu (2002) that for any positive $\epsilon$, we have

$\lim _{m \rightarrow \infty} P\left\{\left|B_{m}(t)-F_{\infty}(t)\right|>\epsilon\right\}=0, \quad t \geqslant 0$,

where $F_{\infty}(t)$, given by

$F_{\infty}(t)=\frac{1-e^{-(\alpha+\beta) t}}{1+(\beta / \alpha) e^{-(\alpha+\beta) t}}$,

is the solution of (3) in a BM with parameters $p=\alpha$ and $q=\beta$. Because (6) is the stochastic analogue of (2), (7) suggests that the BM can be regarded as a model that describes the limiting trajectory of $B_{m}(t)$ in a family of SBMs with the same parameters.

The primary characteristic of interest in an SBM is the expected cumulative number of adoptions $E\left[A_{m}(t)\right]$. In principle, for any finite $m$, this can be derived from standard results for pure birth processes (see Niu 2002, p. 253), but the resulting expression is too complicated to be of practical value. When $m$ is large, this difficulty can be circumvented by a simple approximation. Denote by $F_{m}(t)$ the distribution of the adoption time of a randomly-selected individual in an SBM with specification $(m ; \alpha, \beta)$; then, it immediately follows from (6) that

$E\left[B_{m}(t)\right]=\sum_{j=0}^{m} \frac{j}{m} P\left\{A_{m}(t)=j\right\}=F_{m}(t)$.

Now, as part of the proof of (7), it is also established in Niu (2002) that

$\lim _{m \rightarrow \infty} F_{m}(t)=F_{\infty}(t), \quad t \geqslant 0$.

Hence, from (6), (9), (10), and (8), we have, for sufficiently large $m$,

$E\left[A_{m}(t)\right]=m F_{m}(t) \approx m \frac{1-e^{-(\alpha+\beta) t}}{1+(\beta / \alpha) e^{-(\alpha+\beta) t}}$,

where $\approx$ denotes an approximation.

Of almost-equal interest as the expectation is the variance of the cumulative number of adoptions, $\operatorname{Var}\left[A_{m}(t)\right]$. An exact formula for $\operatorname{Var}\left[A_{m}(t)\right]$ in terms of $F_{m}(t)$ is available in Lemma 4 in Niu (2002); however, as in the case of $E\left[A_{m}(t)\right]$, this formula is intractable for large $m$. An asymptotic analysis of $\operatorname{Var}\left[A_{m}(t)\right]$ is therefore of critical importance. It was conjectured in Niu (2002) that the growth of $\operatorname{Var}\left[A_{m}(t)\right]$ is of order $m$ (as in the case of $\left.E\left[A_{m}(t)\right]\right)$ and $\sqrt{m}\left[B_{m}(t)-F_{\infty}(t)\right]$ is normally distributed as $m$ approaches infinity. Confirmation of this conjecture will allow us to obtain an approximation for $\operatorname{Var}\left[A_{m}(t)\right]$ in the same spirit as (11); furthermore, it will also support the development of a sampling theory for parameter estimation. We now state three asymptotic results for the SBM, in the setting of $D[0, \infty)$, the space of real-valued rightcontinuous functions with left limits (see Billingsley 1999, 
p. 166, or Ethier and Kurtz 1986, p. 116). The first is a functional strong law of large numbers that strengthens (7) to almost sure convergence (uniformly on finite intervals), the second is a functional central limit theorem that verifies asymptotic normality of $\sqrt{m}\left[B_{m}(t)-F_{\infty}(t)\right]$, and the third establishes the convergence of $\operatorname{Var}\left[A_{m}(t)\right] / m$.

We begin with some definitions. From (6), we have that for each $m, B_{m} \equiv\left\{B_{m}(t), t \geqslant 0\right\}$ is a Markov process with state space $E_{m} \equiv\{0 / m, 1 / m, \ldots, m / m\}$; note that state $m / m$ is absorbing and, for $x \in E_{m}-\{m / m\}$, the transition rate of $B_{m}$ from state $x$ to state $x+1 / m$ (see (5)) is given by $m b_{m}(x)$, where, with $\beta_{m} \equiv m \beta /(m-1)$,

$b_{m}(x) \equiv(1-x)\left(\alpha+\beta_{m} x\right)$.

Next, let $V_{m}(t) \equiv \sqrt{m}\left[B_{m}(t)-F_{\infty}(t)\right]$, and define $V_{m} \equiv$ $\left\{V_{m}(t), t \geqslant 0\right\}$. Finally, paralleling $F_{m}(t)=E\left[A_{m}(t)\right] / m$ (see (9)), define

$\phi_{m}(t) \equiv \frac{\operatorname{Var}\left[A_{m}(t)\right]}{m}$,

and note that $\phi_{m}(t)=\operatorname{Var}\left[V_{m}(t)\right]$. Then, we have the following results.

THEOREM 1. The family of processes $\left\{B_{m}\right\}$ can be constructed on a common probability space. On this probability space, we have, for every $t \geqslant 0$,

$\lim _{m \rightarrow \infty} \sup _{0 \leqslant s \leqslant t}\left|B_{m}(s)-F_{\infty}(s)\right|=0$

almost surely.

Theorem 2. As $m \rightarrow \infty$, the family of processes $\left\{V_{m}\right\}$ converges in distribution in $D[0, \infty)$ to a process $V \equiv$ $\{V(t), t \geqslant 0\}$ that satisfies the stochastic differential equation

$d V(t)=b^{\prime}\left(F_{\infty}(t)\right) V(t) d t+\sqrt{b\left(F_{\infty}(t)\right)} d W(t)$,

where, for $x \in E \equiv[0,1]$,

$b(x) \equiv(1-x)(\alpha+\beta x)=\lim _{m \rightarrow \infty} b_{m}(x)$,

$b^{\prime}(\cdot)$ denotes the derivative of $b(\cdot)$, and $\{W(t), t \geqslant 0\}$ is the standard Wiener process (or Brownian motion) with drift 0 and variance rate 1 . The solution of (15) is a Gaussian process, given explicitly by

$V(t)=\int_{0}^{t} \exp \left(\int_{s}^{t} b^{\prime}\left(F_{\infty}(y)\right) d y\right) \sqrt{b\left(F_{\infty}(s)\right)} d W(s)$,

$t \geqslant 0$.

Theorem 3. Let $\psi(t) \equiv \operatorname{Var}[V(t)]$; then, we have

$\psi(t)=F_{\infty}(t)\left[1-F_{\infty}(t)\right]+C(t)$, where

$$
\begin{aligned}
C(t) \equiv & \frac{(1+\beta / \alpha)(\beta / \alpha)\left(1-e^{-(\alpha+\beta) t}\right)}{\left[1+(\beta / \alpha) e^{-(\alpha+\beta) t}\right]^{4} e^{2(\alpha+\beta) t}} \\
& \cdot\left\{2\left[\frac{(\alpha+\beta) t}{1-e^{-(\alpha+\beta) t}}-1\right]+\left(\frac{\beta}{\alpha}\right)\left(1-e^{-(\alpha+\beta) t}\right)\right\} .
\end{aligned}
$$

Furthermore, for every $t \geqslant 0$,

$\lim _{m \rightarrow \infty} \phi_{m}(t)=\psi(t)$.

The proofs of the three theorems are based on very general results for density-dependent jump Markov processes developed in Ethier and Kurtz (1986, Chapter 11). The details are given in the online companion. An intuitive explanation for (15), which can be construed as describing the "velocity of a Brownian particle" (as in the classical Ornstein-Uhlenbeck process; see, e.g., Feller 1971, pp. 335-336) that has time-inhomogeneous acceleration $b^{\prime}\left(F_{\infty}(t)\right) V(t)$ and variance rate $b\left(F_{\infty}(t)\right)$, is also provided there.

The asymptotic variance function (18) has a term-byterm probabilistic interpretation. Let $I_{m j}(t)$ be the indicator function of whether or not the $j$ th adoption has occurred by time $t$; then, $A_{m}(t)=\sum_{j=1}^{m} I_{m j}(t)$. Because a summation is independent of the ordering of the terms, $A_{m}(t)$ can also be represented as

$A_{m}(t)=\sum_{j=1}^{m} I_{m \pi(j)}(t)$,

where $(\pi(1), \pi(2), \ldots, \pi(m))$ is a random permutation of $(1,2, \ldots, m)$. Note that the terms in $(21)$ are now identically distributed, with each determined by the adoption time of a randomly-selected individual in the population. Upon substitution of (21), (13) becomes

$$
\begin{aligned}
\phi_{m}(t)= & \frac{1}{m} \sum_{j=1}^{m} \operatorname{Var}\left[I_{m \pi(j)}(t)\right] \\
& +\frac{1}{m} \sum_{\substack{k=1 \\
k \neq l}}^{m} \sum_{\substack{l=1 \\
k \neq l}}^{m} \operatorname{Cov}\left[I_{m \pi(k)}(t), I_{m \pi(l)}(t)\right] .
\end{aligned}
$$

By conditioning on $A_{m}(t)$ (similar to (9)), we have $E\left[I_{m \pi(j)}(t)\right]=F_{m}(t)$ for all $j$, and hence

$$
\begin{aligned}
\operatorname{Var}\left[I_{m \pi(j)}(t)\right] & =E\left[I_{m \pi(j)}(t)\right]\left[1-E\left[I_{m \pi(j)}(t)\right]\right] \\
& =F_{m}(t)\left[1-F_{m}(t)\right] .
\end{aligned}
$$

It then follows from (10) that

$\lim _{m \rightarrow \infty} \frac{1}{m} \sum_{j=1}^{m} \operatorname{Var}\left[I_{m \pi(j)}(t)\right]=F_{\infty}(t)\left[1-F_{\infty}(t)\right]$, 
and this shows that the first term in (18) originates from the variance of $I_{m \pi(j)}(t)$. Moreover, a comparison of (22) and (23) with (20) and (18) shows that

$$
\lim _{m \rightarrow \infty} \frac{1}{m} \sum_{\substack{k=1 \\ k \neq l}}^{m} \sum_{l=1}^{m} \operatorname{Cov}\left[I_{m \pi(k)}(t), I_{m \pi(l)}(t)\right]=C(t) ;
$$

thus, the second term in (18) originates from the (common) pairwise covariances between the $I_{m \pi(j)}(t)$ s. Furthermore, it is easily shown that

$\lim _{\alpha \rightarrow 0} \frac{C(t)}{F_{\infty}(t)\left[1-F_{\infty}(t)\right]}=e^{\beta t}-1$.

Hence, for a positive $t$, the cross-covariances term $C(t)$ (which is bell shaped with $\lim _{t \rightarrow 0} C(t)=\lim _{t \rightarrow \infty} C(t)=0$ ) dominates the variances term $F_{\infty}(t)\left[1-F_{\infty}(t)\right]$ when $0 \approx$ $\alpha \ll \beta$ (the case for most successful products).

An immediate consequence of Theorems 2 and 3 is the following (one-dimensional) central limit theorem.

Corollary 1. As $m \rightarrow \infty$, we have, for every $t \geqslant 0$,

$\frac{A_{m}(t)-m F_{\infty}(t)}{\sqrt{m \psi(t)}} \Rightarrow N(0,1)$,

where $\Rightarrow$ denotes convergence in distribution and $N(0,1)$ denotes a standard normal random variable with mean 0 and variance 1.

It follows from (24) that when $m$ is large, the distribution of $A_{m}(t)$ can be accurately approximated by that of a normal random variable with mean $m F_{\infty}(t)$ and variance $m \psi(t)$. The convergences of $F_{m}(t)$ and $\phi_{m}(t)$ to $F_{\infty}(t)$ and $\psi(t)$, respectively, are illustrated in Figures 1 and 2 . Note that, like the density curve $f_{\infty}(t) \equiv d F_{\infty}(t) / d t$, the asymptotic variance function $\psi(t)$ is bell shaped. Corollary 1 will be instrumental in the model development in $\S 3$.

Figure 1. Convergence of $F_{m}(t)$ to $F_{\infty}(t)$.

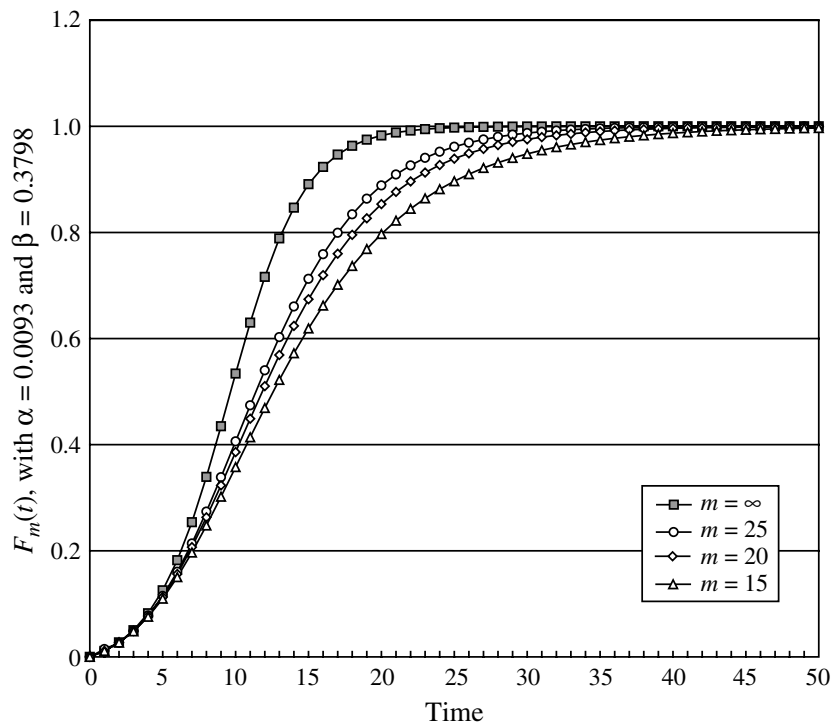

Figure 2. Convergence of $\phi_{m}(t)$ to $\psi(t)$.

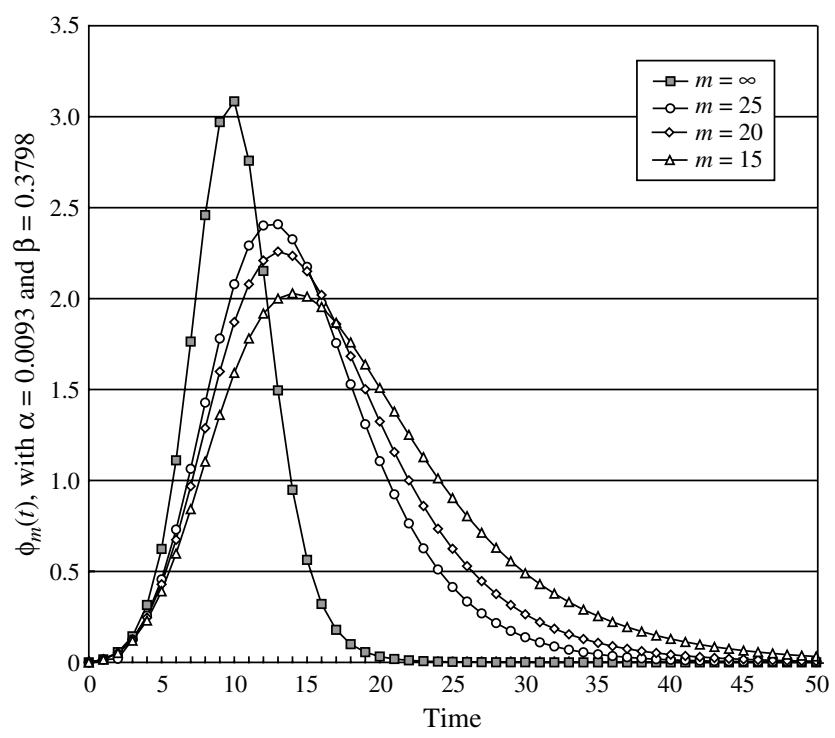

\subsection{A Piecewise Stochastic Bass Model}

Let $0 \equiv t_{0}<t_{1}<t_{2}<\cdots<t_{n}<\infty$ be a sequence of time epochs at which incremental-sales data of a product are to be observed. These epochs (not necessarily equally spaced) partition the time interval $\left[t_{0}, t_{n}\right]$ into $n$ periods. For $1 \leqslant$ $i \leqslant n$, let $A_{i}$ be the total adoptions/sales in the $i$ th period. In the Piecewise Stochastic Bass Model (PSBM), we will formulate the $A_{i} \mathrm{~s}$ as adoption counts in a history-dependent sequence of SBMs with varying specifications. As before, let $m$ be the size of the target population, and let each individual in this population have an intrinsic adoption rate $\alpha$ and an induction rate $\beta$. The value of $m$ is assumed to be constant over time; and the rates $\alpha$ and $\beta$ are assumed to be constant both over time and across individuals.

Note that we have set $t_{0}$ to 0 . This, however, does not necessarily mean that the product is launched at time 0 . Let $t_{-1}$ be the epoch at which the product is introduced; then, we assume that $t_{-1} \leqslant t_{0}$ and that the total sales during $\left[t_{-1}, t_{0}\right]$ is given by $a_{0}$. In practice, $a_{0}$ will come from historical data, which we assume is available. Adopters counted in $a_{0}$ can be construed as "innovators" (or early adopters, i.e., this is a relative terminology), in the language of Bass (1969) and Rogers (1995).

We now consider $\left[t_{0}, t_{1}\right]$, the first time period, and introduce an important point of departure from the SBM. We will assume that of the $m-a_{0}$ potential adopters at $t_{0}$, only $\left(m-a_{0}\right) \pi_{1}$ individuals, where $\pi_{1}$ is a specified fraction, are genuine prospects for actual adoption/purchase during $\left[t_{0}, t_{1}\right]$. The remaining $\left(m-a_{0}\right)\left(1-\pi_{1}\right)$ individuals are considered dormant, in that they are not yet ready to engage in product purchase. The fraction $\pi_{1}$ is intended to capture the aggregated effect of available supply of the product, reach of distribution channels, level of product quality, price, reach and impact of promotion/information (e.g., of advertising), and any other factors 
that contribute to the readiness of a potential adopter. We shall refer to $\pi_{1}$ as the participation fraction for period 1 , and to individuals counted in $\left(m-a_{0}\right) \pi_{1}$ as ready potential adopters. If $\left(m-a_{0}\right) \pi_{1}$ is not an integer, we interpret it to mean $\left\lfloor\left(m-a_{0}\right) \pi_{1}\right\rfloor$; that is, we round it down. This convention will be adopted throughout to avoid notational complication.

It follows that the adoption process beyond time $t_{0}$ is the "continuation" of an SBM (with original specification $(m ; \alpha, \beta))$ in which $a_{0}$ individuals have already bought, but now only $\left(m-a_{0}\right) \pi_{1}$ out of the remaining $m-a_{0}$ potential adopters are ready for product purchase. Because each of the existing adopters makes a contact with each of the potential adopters at rate $\beta /(m-1)$, the memoryless property of exponential random variables implies that the distribution of time to first purchase in $\left[t_{0}, t_{1}\right]$ is exponential with rate $\left(m-a_{0}\right) \pi_{1}\left[\alpha+(\beta /(m-1)) a_{0}\right]$. Similarly, because one of the ready potential adopters switches into an existing adopter at the time of first purchase, we also have that the next inter-purchase time is exponentially distributed with rate

$\left[\left(m-a_{0}\right) \pi_{1}-1\right]\left[\left(\alpha+\frac{\beta}{m-1} a_{0}\right)+\frac{\beta}{m-1}\right]$,

and continuation of this argument shows that in general, for any $k \geqslant 1$, the elapsed time between the $k$ th and the $(k+1)$ th purchase in $\left[t_{0}, t_{1}\right]$ is exponentially distributed with rate

$\left[\left(m-a_{0}\right) \pi_{1}-k\right]\left[\left(\alpha+\frac{\beta}{m-1} a_{0}\right)+\frac{\beta}{m-1} k\right]$.

Observe that the last expression can be rewritten as

$\left[\left(m-a_{0}\right) \pi_{1}-k\right]\left[\alpha_{1}+\frac{\beta_{1}}{\left(m-a_{0}\right) \pi_{1}-1} k\right]$,

where

$\alpha_{1} \equiv \alpha+\frac{\beta}{m-1} a_{0}$

and

$\beta_{1} \equiv \frac{\left(m-a_{0}\right) \pi_{1}-1}{m-1} \beta$.

A comparison of (25) with (5) then yields the result that increments of the adoption process in $\left[t_{0}, t_{1}\right]$ are stochastically identical to that in a "new" SBM that starts from state 0 at $t_{0}$, with population size $\left(m-a_{0}\right) \pi_{1}$, intrinsic adoption rate $\alpha_{1}$, and induction rate $\beta_{1}$. In other words, the adoption count $A_{1}$ in $\left[t_{0}, t_{1}\right]$ can be taken as a realization of $A_{\left(m-a_{0}\right) \pi_{1}}\left(t_{1}-t_{0}\right)$ in an SBM with specification $\left(\left(m-a_{0}\right) \pi_{1} ; \alpha_{1}, \beta_{1}\right)$. The change-of-parameters formulas (26) and (27) (and other ones to follow) explicitly formalize the intuitive concept that as we advance time from one period to the next, the diffusion rates should be properly "updated" to reflect both the growing influence from the accumulation of existing adopters and the dynamicallychanging population size.

The remaining $A_{i} \mathrm{~s}$ can now be constructed recursively in a similar manner. Consider the $i$ th period $\left[t_{i-1}, t_{i}\right]$ for any $2 \leqslant i \leqslant n$. Observe first that at epoch $t_{i-1}$, there are $a_{0}+\sum_{j=1}^{i-1} A_{j}$ existing adopters. A repeat of the above argument then shows that for any given history of realizations $A_{1}=a_{1}, \ldots, A_{i-1}=a_{i-1}$, we can, conditionally, define $A_{i}$ as the adoption count that results from a run of duration $t_{i}-t_{i-1}$ in a new SBM that starts at time $t_{i-1}$ with specification $\left(\left[m-\left(a_{0}+\sum_{j=1}^{i-1} a_{j}\right)\right] \pi_{i} ; \alpha_{i}, \beta_{i}\right)$, where $\pi_{i}$ is the participation fraction for period $i$,

$\alpha_{i} \equiv \alpha+\frac{\beta}{m-1}\left(a_{0}+\sum_{j=1}^{i-1} a_{j}\right)$,

and

$\beta_{i} \equiv \frac{\left[m-\left(a_{0}+\sum_{j=1}^{i-1} a_{j}\right)\right] \pi_{i}-1}{m-1} \beta$.

Finally, iterating these definitions sequentially from period 2 through period $n$ yields a discrete-time Markov process $\left\{A_{i}, i=1, \ldots, n\right\}$ that we shall henceforth refer to as a PSBM with specification $\left(m, a_{0} ;\left\{\pi_{i}\right\}_{i=1}^{n}, \alpha, \beta\right)$. Note that if $a_{0}=0$ and $\pi_{i}=1$ for all $1 \leqslant i \leqslant n$, then this PSBM specializes (see (25)-(29)) to a corresponding discretetime process that describes successive incremental adoptions during $\left[t_{0}, t_{n}\right]$ in an ordinary SBM with specification $(m ; \alpha, \beta)$.

The construction of the PSBM above also naturally suggests a useful approximation scheme for the expected values of the $A_{i} \mathrm{~s}$, which we develop next.

For $1 \leqslant i \leqslant n$, define $\mu_{i} \equiv E\left[A_{i}\right]$; then, the scheme proceeds recursively, starting with an approximation for $\mu_{1}$. Suppose that $m$ is sufficiently large. Because $A_{1}$ is distributed as $A_{\left(m-a_{0}\right) \pi_{1}}\left(t_{1}-t_{0}\right)$ in a new SBM with specification $\left(\left(m-a_{0}\right) \pi_{1} ; \alpha_{1}, \beta_{1}\right)$, it follows from (11) that $\mu_{1} \approx \hat{\mu}_{1}$, where

$\hat{\mu}_{1} \equiv\left(m-a_{0}\right) \pi_{1} \frac{1-e^{-\left(\alpha_{1}+\beta_{1}\right)\left(t_{1}-t_{0}\right)}}{1+\left(\beta_{1} / \alpha_{1}\right) e^{-\left(\alpha_{1}+\beta_{1}\right)\left(t_{1}-t_{0}\right)}}$.

Next, observe that from (13), (20), and (11), the coefficient of variation of $A_{m}(t)$ is approximately equal to $\sqrt{m \psi(t)} /\left[m F_{\infty}(t)\right]$, which is of order $1 / \sqrt{m}$. Hence, we can "pretend" that $\hat{\mu}_{1}$ is an actual realization of $A_{1}$. By substituting $\hat{\mu}_{1}$ as $a_{1}$ in (28) and (29), we then have the approximations

$\alpha_{2} \approx \hat{\alpha}_{2} \equiv \alpha+\frac{\beta}{m-1}\left(a_{0}+\hat{\mu}_{1}\right)$

and

$\beta_{2} \approx \hat{\beta}_{2} \equiv \frac{\left[m-\left(a_{0}+\hat{\mu}_{1}\right)\right] \pi_{2}-1}{m-1} \beta$. 
It follows that $A_{2}$ is approximately distributed as the adoption count that results from a run of duration $t_{2}-t_{1}$ in a new SBM with specification $\left(\left[m-\left(a_{0}+\hat{\mu}_{1}\right)\right] \pi_{2} ; \hat{\alpha}_{2}, \hat{\beta}_{2}\right)$. Therefore, from another application of (11), we have $\mu_{2} \approx \hat{\mu}_{2}$, where

$\hat{\mu}_{2} \equiv\left[m-\left(a_{0}+\hat{\mu}_{1}\right)\right] \pi_{2} \frac{1-e^{-\left(\hat{\alpha}_{2}+\hat{\beta}_{2}\right)\left(t_{2}-t_{1}\right)}}{1+\left(\hat{\beta}_{2} / \hat{\alpha}_{2}\right) e^{-\left(\hat{\alpha}_{2}+\hat{\beta}_{2}\right)\left(t_{2}-t_{1}\right)}}$.

Continuation of the same scheme then yields an entire sequence of approximations $\left\{\hat{\mu}_{i}, i=1, \ldots, n\right\}$ for the $\mu_{i} \mathrm{~s}$. Formally, for $1 \leqslant i \leqslant n$, the $\hat{\mu}_{i}$ s are determined by the following set of recursions (ill-defined sums are interpreted as 0 throughout):

$$
\begin{aligned}
& \hat{\alpha}_{i} \equiv \alpha+\frac{\beta}{m-1}\left(a_{0}+\sum_{j=1}^{i-1} \hat{\mu}_{j}\right), \\
& \hat{\beta}_{i} \equiv \frac{\left[m-\left(a_{0}+\sum_{j=1}^{i-1} \hat{\mu}_{j}\right)\right] \pi_{i}-1}{m-1} \beta,
\end{aligned}
$$

and

$\hat{\mu}_{i} \equiv\left[m-\left(a_{0}+\sum_{j=1}^{i-1} \hat{\mu}_{j}\right)\right] \pi_{i} \frac{1-e^{-\left(\hat{\alpha}_{i}+\hat{\beta}_{i}\right)\left(t_{i}-t_{i-1}\right)}}{1+\left(\hat{\beta}_{i} / \hat{\alpha}_{i}\right) e^{-\left(\hat{\alpha}_{i}+\hat{\beta}_{i}\right)\left(t_{i}-t_{i-1}\right)}}$.

The approximations (31)-(33) will play an instrumental role in $\$ 3.2$.

\section{The Piecewise-Diffusion Model}

While the PSBM is designed to model individual-level stochastic behavior of adopters, it is not realistic to expect any model to fully explain the fluctuations observed in actual sales data. It is therefore important to further expand the PSBM to include explicit components that reflect the aggregate impacts of unaccounted exogenous influences (see, e.g., Srinivasan and Mason 1986, pp. 170-171). In this section, we develop two such formulations, an actual-history version and an expected-history version; and we shall refer to both as the Piecewise-Diffusion Model (PDM). In addition to the incorporation of exogenous errors, the central limit theorem (24) will now be integrated into these formulations.

\subsection{The Actual-History PDM}

Consider, as in $\$ 2.2, n$ consecutive time periods; and let $S_{i}$ be the total sales of a product in period $i$. We assume that each $S_{i}$ can be modeled as the sum of two independent variables:

$S_{i} \sim A_{i}+D_{i}$

where $\sim$ denotes equality in distribution. The sequences of $A_{i} \mathrm{~s}$ and $D_{i} \mathrm{~s}$ are formulated as follows.
The $D_{i} \mathrm{~s}$ are intended to capture errors due to both exogenous disturbance and model misspecification/approximations. Specifically, we assume that $D_{i}$ is normally distributed with mean 0 and variance $\delta_{i}^{2} \equiv \delta^{2}\left(t_{i}-t_{i-1}\right)$, where $\delta^{2}$ is a constant variance rate. We also assume that the $D_{i} \mathrm{~s}$ are independent.

The $A_{i} \mathrm{~s}$ are intended to model the successive numbers of adoptions in a PSBM. However, because the PSBM is dynamic, we need to slightly modify the earlier definitions to reflect dependence on the history of the $S_{i}$ s (which includes that of the $D_{i} \mathrm{~s}$ ), rather than of the $A_{i} \mathrm{~s}$ alone. Most importantly, we further assume that $m$ is sufficiently large, so that we can approximate the distribution of the modified $A_{i}$ s via (24).

Specifically, in our first formulation, the $A_{i} \mathrm{~s}$ are recursively constructed based on the history of actual sales; and this is done as follows. For period one, recall from $\$ 2.2$ that $A_{1}$ is distributed as $A_{\left(m-a_{0}\right) \pi_{1}}\left(t_{1}-t_{0}\right)$ in a new SBM with specification $\left(\left(m-a_{0}\right) \pi_{1} ; \alpha_{1}, \beta_{1}\right)$. Let $\psi_{1}(t)$ be a version of (18) in which $\alpha$ and $\beta$ are replaced, respectively, by $\alpha_{1}$ and $\beta_{1}$. Then, because $E\left[A_{1}\right]=\mu_{1} \approx \hat{\mu}_{1}$, where $\hat{\mu}_{1}$ is given by (30), it follows from (24) that we have, approximately,

$A_{1} \sim \hat{\mu}_{1}+\widehat{V}_{1}$,

where

$\widehat{V}_{1} \sim N\left(0,\left(m-a_{0}\right) \pi_{1} \psi_{1}\left(t_{1}-t_{0}\right)\right)$

Next, consider any $i$, where $2 \leqslant i \leqslant n$, and let $S_{1}=$ $s_{1}, \ldots, S_{i-1}=s_{i-1}$ be any sequence of realizations of $S_{1}, \ldots, S_{i-1}$. Then, again according to (24), we have the (conditional) approximation

$$
\begin{aligned}
A_{i} \sim & {\left[m-\left(a_{0}+\sum_{j=1}^{i-1} s_{j}\right)\right] } \\
& \cdot \pi_{i} \frac{1-e^{-\left(\alpha_{i}+\beta_{i}\right)\left(t_{i}-t_{i-1}\right)}}{1+\left(\beta_{i} / \alpha_{i}\right) e^{-\left(\alpha_{i}+\beta_{i}\right)\left(t_{i}-t_{i-1}\right)}}+\widehat{V}_{i},
\end{aligned}
$$

where $\alpha_{i}$ and $\beta_{i}$ are versions of (28) and (29) in which $a_{j}$ is replaced by $s_{j}$ and, with $\psi_{i}(t)$ defined similar to $\psi_{1}(t)$,

$\widehat{V}_{i} \sim N\left(0,\left[m-\left(a_{0}+\sum_{j=1}^{i-1} s_{j}\right)\right] \pi_{i} \psi_{i}\left(t_{i}-t_{i-1}\right)\right)$.

Now, for $1 \leqslant i \leqslant n$, let

$\hat{s}_{i} \equiv\left[m-\left(a_{0}+\sum_{j=1}^{i-1} s_{j}\right)\right] \pi_{i} \frac{1-e^{-\left(\alpha_{i}+\beta_{i}\right)\left(t_{i}-t_{i-1}\right)}}{1+\left(\beta_{i} / \alpha_{i}\right) e^{-\left(\alpha_{i}+\beta_{i}\right)\left(t_{i}-t_{i-1}\right)}}$

and

$\hat{\sigma}_{i} \equiv\left\{\left[m-\left(a_{0}+\sum_{j=1}^{i-1} s_{j}\right)\right] \pi_{i} \psi_{i}\left(t_{i}-t_{i-1}\right)+\delta_{i}^{2}\right\}^{1 / 2}$, 
and let $\left\{Z_{i}, i=1, \ldots, n\right\}$ be a sequence of i.i.d. $N(0,1)$ random variables. Then, (34) and the recursions in (35)-(38) together define a discrete-time Markov process $\left\{S_{i}, i=1, \ldots, n\right\}$, where

$S_{i} \sim \hat{s}_{i}+\hat{\sigma}_{i} Z_{i}$,

that models incremental sales of the product over the given $n$ periods. We shall refer to this process as an actualhistory PDM with specification $\left(m, a_{0} ;\left\{\pi_{i}\right\}_{i=1}^{n}, \alpha, \beta, \delta\right)$. The description "piecewise-diffusion" is adopted because, for each $i$, the normalized $A_{i}$, i.e.,

$$
\frac{A_{i}-E\left[A_{i}\right]}{\left\{\left[m-\left(a_{0}+\sum_{j=1}^{i-1} s_{j}\right)\right] \pi_{i}\right\}^{1 / 2}}
$$

(for given $S_{1}=s_{1}, \ldots, S_{i-1}=s_{i-1}$ ), corresponds to the outcome of a run of a diffusion process (an instance of $V$ in Theorem 2, more precisely) for a duration of length $t_{i}-t_{i-1}$.

\subsection{The Expected-History PDM}

Recall that in \$2.2, we developed an approximation scheme for the expected incremental adoptions in a PSBM. This was given in recursions (31)-(33). In this section, we show that (33) can be incorporated into the construction of the $A_{i} \mathrm{~s}$ in $\$ 3.1$ to yield a second version of the PDM that is based on past expected sales, as opposed to past actual sales.

We will, again, assume that (34) holds. Next, note that because $E\left[D_{i}\right]=0$, we have $E\left[S_{i}\right]=E\left[A_{i}\right]$ for all $1 \leqslant i \leqslant n$. This suggests that we can approximate the actual sales $s_{j}$ in (37) and (38) by $E\left[A_{j}\right]$. Because $E\left[A_{j}\right]=\mu_{j} \approx \hat{\mu}_{j}$, given by (33), the resulting approximation is

$$
\begin{aligned}
A_{i} \sim & {\left[m-\left(a_{0}+\sum_{j=1}^{i-1} \hat{\mu}_{j}\right)\right] } \\
& \cdot \pi_{i} \frac{1-e^{-\left(\hat{\alpha}_{i}+\hat{\beta}_{i}\right)\left(t_{i}-t_{i-1}\right)}}{1+\left(\hat{\beta}_{i} / \hat{\alpha}_{i}\right) e^{-\left(\hat{\alpha}_{i}+\hat{\beta}_{i}\right)\left(t_{i}-t_{i-1}\right)}}+\widehat{V}_{i},
\end{aligned}
$$

where $\hat{\alpha}_{i}$ and $\hat{\beta}_{i}$ are given by (31) and (32) and, with $\psi_{i}(t)$ being a version of (18) in which $\alpha$ and $\beta$ are replaced by $\hat{\alpha}_{i}$ and $\hat{\beta}_{i}$,

$\widehat{V}_{i} \sim N\left(0,\left[m-\left(a_{0}+\sum_{j=1}^{i-1} \hat{\mu}_{j}\right)\right] \pi_{i} \psi_{i}\left(t_{i}-t_{i-1}\right)\right)$.

Finally, from (34), (42), and (43), we again arrive at (41), but now with (39) and (40) revised to

$$
\begin{aligned}
\hat{s}_{i}=\hat{\mu}_{i}= & {\left[m-\left(a_{0}+\sum_{j=1}^{i-1} \hat{\mu}_{j}\right)\right] } \\
& \cdot \pi_{i} \frac{1-e^{-\left(\hat{\alpha}_{i}+\hat{\beta}_{i}\right)\left(t_{i}-t_{i-1}\right)}}{1+\left(\hat{\beta}_{i} / \hat{\alpha}_{i}\right) e^{-\left(\hat{\alpha}_{i}+\hat{\beta}_{i}\right)\left(t_{i}-t_{i-1}\right)}}
\end{aligned}
$$

and

$\hat{\sigma}_{i}=\left\{\left[m-\left(a_{0}+\sum_{j=1}^{i-1} \hat{\mu}_{j}\right)\right] \pi_{i} \psi_{i}\left(t_{i}-t_{i-1}\right)+\delta_{i}^{2}\right\}^{1 / 2}$.

We shall refer to the discrete-time Markov process $\left\{S_{i}, i=\right.$ $1, \ldots, n\}$ defined by (41), (44), and (45) as an expectedhistory PDM with specification $\left(m, a_{0} ;\left\{\pi_{i}\right\}_{i=1}^{n}, \alpha, \beta, \delta\right)$.

It is important to note that both $\left\{\hat{s}_{i}, i=1, \ldots, n\right\}$ and $\left\{\hat{\sigma}_{i}, i=1, \ldots, n\right\}$ are fully determined once the specification $\left(m, a_{0} ;\left\{\pi_{i}\right\}_{i=1}^{n}, \alpha, \beta, \delta\right)$ is given. Therefore, the expected-history PDM can be viewed as a heteroscedastic nonlinear regression model.

\section{Parameter Formulations}

In this section, we show that the PDM is a highly-flexible framework that can be specialized to yield multiple model regimes by designating different components of its specification $\left(m, a_{0} ;\left\{\pi_{i}\right\}_{i=1}^{n}, \alpha, \beta, \delta\right)$ as parameters. In particular, we will develop parameter formulations that progressively incorporate the effects of price and advertising. Because in practice the data collection intervals are of equal length, typically one year, we will henceforth assume that $t_{i}=i$ and therefore $t_{i}-t_{i-1}=1$ for all $1 \leqslant i \leqslant n$; this also implies that $\delta_{i}^{2}=\delta^{2}$ for all $i$.

Note that for any parameter formulation, the associated likelihood function of the PDM can be constructed easily. Consider the actual-history PDM and suppose that a particular sequence of sales $\left\{s_{i}, i=1, \ldots, n\right\}$ is given. From (41), we have that, for any $i$ and any given $S_{1}=s_{1}, \ldots, S_{i-1}=$ $s_{i-1}$, the conditional likelihood of observing $S_{i}=s_{i}$ is

$\frac{1}{\sqrt{2 \pi} \hat{\sigma}_{i}} \exp \left\{-\frac{1}{2}\left(\frac{s_{i}-\hat{s}_{i}}{\hat{\sigma}_{i}}\right)^{2}\right\}$

where $\hat{s}_{i}$ and $\hat{\sigma}_{i}$ are given by (39) and (40), respectively. Taking iterative products of the conditional densities (46) over $i$ then yields the likelihood function associated with the entire sequence $\left\{s_{i}, i=1, \ldots, n\right\}$ :

$L\left(m, a_{0} ;\left\{\pi_{i}\right\}_{i=1}^{n}, \alpha, \beta, \delta\right) \equiv \prod_{i=1}^{n} \frac{1}{\sqrt{2 \pi} \hat{\sigma}_{i}} \exp \left\{-\frac{1}{2}\left(\frac{s_{i}-\hat{s}_{i}}{\hat{\sigma}_{i}}\right)^{2}\right\}$.

This leads, after ignoring a constant term, to the log-likelihood function

$$
\begin{aligned}
& \ln L\left(m, a_{0} ;\left\{\pi_{i}\right\}_{i=1}^{n}, \alpha, \beta, \delta\right) \\
& \equiv-\sum_{i=1}^{n} \ln \hat{\sigma}_{i}-\frac{1}{2} \sum_{i=1}^{n}\left(\frac{s_{i}-\hat{s}_{i}}{\hat{\sigma}_{i}}\right)^{2} .
\end{aligned}
$$

The corresponding log-likelihood function for the expectedhistory PDM is also given by (47), but with (44) and (45) replacing (39) and (40), respectively. 


\subsection{The SBM and Its Relation to the BM}

As noted in $\$ 2.2$, the PSBM reduces to an SBM with specification $(m ; \alpha, \beta)$ if we let $a_{0}=0$ and $\pi_{i}=1$ for all $1 \leqslant$ $i \leqslant n$. Clearly, the discrete-time process defined by (41) can also be reduced in the same manner to an empirical operationalization of the SBM with corresponding specification $(m ; \alpha, \beta, \delta)$; to simplify language, we will refer to this empirical version of the SBM as SBM as well. Historically, the parameters in the BM have been taken to be $m, p$, and $q$; we will therefore follow this tradition in the SBM and let all four components of the specification $(m ; \alpha, \beta, \delta)$ be parameters. These parameters can be estimated from data, using (47).

In light of (11), it is natural to expect the successive expected incremental sales in the SBM to be close to the discretized version of the sales trajectory in the corresponding BM. That is,

$E\left[A_{i}\right] \approx m\left[F_{\infty}\left(t_{i}\right)-F_{\infty}\left(t_{i-1}\right)\right]$.

This can be supported by an informal argument; details are given in the online companion.

Observe that with the introduction of an independent sequence of i.i.d. exogenous errors, (48) becomes the empirical operationalization of the BM in Srinivasan and Mason (1986). Note, however, that in addition to the means in (48), the SBM provides, in (40) or (45), information on the variability of the sales trajectory (aside from the exogenous error variance $\delta^{2}$ ); further discussion appears at the end of $\S 5$.

\subsection{The Basic PDM}

Consider an actual-history (say) PDM with specification $\left(m, a_{0} ;\left\{\pi_{i}\right\}_{i=1}^{n}, \alpha, \beta, \delta\right)$. Recall that in each period of the PDM, we have an SBM. This suggests that if we define for $1 \leqslant i \leqslant n$,

$m_{i} \equiv\left[m-\left(a_{0}+\sum_{j=1}^{i-1} S_{j}\right)\right] \pi_{i}+\left(a_{0}+\sum_{j=1}^{i-1} S_{j}\right)$,

then $m_{i}$ can be construed as the applicable "market ceiling" during period $i$. In other words, if we focus attention on period $i$ alone, then $m_{i}$ plays the same role as " $m$ " in a stochastically equivalent SBM that has accumulated $a_{0}+$ $\sum_{j=1}^{i-1} S_{j}$ adopters by time $t_{i-1}$.

Observe, however, that there is a technical distinction between $m_{i}$ in the PDM and $m$ in the SBM/BM, namely, that in the former setting, if $a_{0}$ and a history of sales $S_{1}, \ldots, S_{i-1}$ are given, then $m_{i}$ depends on both $m$ and $\pi_{i}$. Clearly, this implies that when we attempt to infer the value of $m_{i}$ empirically, a degree of redundancy exists if we allow both $m$ and $\pi_{i}$ to freely vary in (49). Therefore, except for the parameter regime of an SBM in $\S 4.1$ (where $\pi_{i}=1$ for all $i$ ), we will henceforth not consider $m$ as a parameter in the PDM. With $m$ fixed, and with the sales history given, there is then a one-to-one correspondence between the $m_{i} \mathrm{~s}$ and the $\pi_{i} \mathrm{~s}$.

That we do not designate $m$ as a parameter, of course, does not mean that the value of $m$ can be chosen arbitrarily. For household products, a natural specification is to let $m$ equal the total number of households in the last period of study (Schmittlein and Mahajan 1982, p. 66; Jain and Rao 1990, Model 1). For personal products, such as cellular phones, we could take $m$ to be twice that for household products. Thus, there also is a conceptual distinction between $m$ in the PDM and $m$ in the SBM/BM, namely, that in the former it should be in the general neighborhood of the "factual" ultimate market ceiling over all time periods. As such, the value of $m$ is more or less generic in that it is not expected to be very sensitive to the detailed nature of a particular product. Regardless of the proposed rationale for a particular broad type of products, a basic requirement is that $m$ be set at an appropriately-high level, so that it does not constrain, or interfere with, the ability of the $\pi_{i} \mathrm{~s}$ to produce a reasonable range of values for the $m_{i} \mathrm{~s}$, which, as formulated in (49), are the time-dependent and product-specific market ceilings (or penetration levels) that we truly intend to infer.

We now make our first attempt at the important task of formulating the $\pi_{i} \mathrm{~s}$. As noted in $\$ 2.2$, the $\pi_{i}$ s are intended to reflect the aggregated effects of factors that contribute to the readiness of potential adopters in individual time periods. A common difficulty in practice is that although we do have a good sense as to which factors (e.g., marketingmix variables) might be of significant relevance, we may not have data, or even simple metrics, on the movements of specific contributing factors. This difficulty, in fact, is always present if we are at the prelaunch or newly-launched stage of a product. Thus, it is highly desirable to have a simplified model regime in which the $\pi_{i} \mathrm{~s}$ are assumed to be at a constant base level over time; and this, formally, means setting $\pi_{i}=\pi$ (where $0<\pi \leqslant 1$ ) for all $1 \leqslant i \leqslant n$ in the PDM. We shall refer to this backbone special case, with reduced specification $\left(m, a_{0} ; \pi, \alpha, \beta, \delta\right)$, as the basic PDM. Note that as a consequence of our discussion above, only four parameters (as in the case of $\mathrm{SBM} / \mathrm{BM}$ ) are to be estimated from data in the basic PDM, namely, $\pi, \alpha, \beta$, and $\delta$; the values of $m$ and $a_{0}$ are assumed given.

\subsection{The Full PDM with Price and Advertising}

In this section, we proceed beyond the basic PDM by proposing parameter formulations that include price and advertising. This is done sequentially, first price and then advertising.

The formulations below are developed via a comprehensive set of experimentations (details of which are voluminous and hence are omitted) based on actual data; that is, within the context of PDM, they have been found to be the "most" consistent combinations across multiple products. In addition to experimental calibration, key features of the proposed formulations are also supported 
theoretically/conceptually by common-sense intuitions; and these will be explained along with the formulations.

4.3.1. The Price-Response Function. The basic premise we make is that prices primarily affect the residual market potentials in different time periods via the $\pi_{i} \mathrm{~s}$. This premise is similar in spirit to Model 3 in Jain and Rao (1990, p. 165, Equation (2.14)). Specifically, let $\pi(p)$, where $p \geqslant 0$, be a "response" function that prescribes the participation fraction in force in the PDM when price equals $p$; and let $p_{\text {ref }}$ be a given reference price. Then, we propose that $\pi(p)$ has the following functional form:

$$
\pi(p)=\pi_{m}\left\{1-\exp \left\{-\left[-\ln \left(1-\frac{\pi}{\pi_{m}}\right)\right]\left(\frac{p}{p_{\text {ref }}}\right)^{-\eta}\right\}\right\}
$$

where $\pi_{m}, \pi$, and $\eta$, with $0<\pi \leqslant \pi_{m} \leqslant 1$ and $\eta \geqslant 0$, are three parameters that jointly control the shape of $\pi(p)$. The formulation in (50) is designed to capture a host of desirable properties. In particular, it mirrors a variety of intuitive beliefs regarding the price response of consumers. Details are now discussed below. Note that when $\pi=\pi_{m}$ or $\eta=0,(50)$ yields a constant. To avoid unnecessary complications, we will assume that $\pi<\pi_{m}$ and $\eta>0$ in the ensuing discussion.

Clearly, $\pi(p)$ is decreasing in $p$. If we define

$\kappa \equiv-\ln \left(1-\frac{\pi}{\pi_{m}}\right)$

then (50) assumes the simpler-looking form

$$
\pi(p)=\pi_{m}\left\{1-\exp \left\{-\kappa\left(\frac{p}{p_{\text {ref }}}\right)^{-\eta}\right\}\right\} .
$$

Hence, for any given pair of $\pi_{m}$ and $\eta$, the value of $\kappa$ "controls" the rate at which $\pi(p)$ declines (also see (54) below). Note further that if $\pi_{m}=1$ and $p_{\text {ref }}=1$, then (52) becomes $\pi(p)=1-\exp \left(-\kappa p^{-\eta}\right)($ where $\kappa=-\ln (1-\pi))$, which can be construed as the primitive form of the priceresponse function (50).

For $1 \leqslant i \leqslant n$, let $p_{i}$ be the average price of the product during period $i$; and, as a natural convention, let $p_{\text {ref }}=p_{1}$. Suppose that the entire set of prices $\left\{p_{i}, i=1, \ldots, n\right\}$ is available. Then, according to (50), we have $\pi_{i}=\pi\left(p_{i}\right)$ for $1 \leqslant i \leqslant n ;$ that is,

$\pi_{i}=\pi_{m}\left\{1-\exp \left\{-\left[-\ln \left(1-\frac{\pi}{\pi_{m}}\right)\right]\left(\frac{p_{i}}{p_{1}}\right)^{-\eta}\right\}\right\}$.

The convention $p_{\text {ref }}=p_{1}$ means that price responses in (53) are based on the "normalized" prices $\left\{p_{i} / p_{1}\right\}_{i=1}^{n}$. Note that if $p_{i}=p_{1}$ for all $1 \leqslant i \leqslant n$, then $\pi_{i}=\pi$ for all $i$, and we recover the basic PDM. A desirable fact in this case is that the actual value of $p_{1}$ is irrelevant. Therefore, recovery of the basic PDM is designed to be independent of the initial price level. This feature is helpful in maintaining parameter consistency between PDMs with and without price when one conducts empirical analysis. It is also potentially helpful when we compare parameter values for different products.

Clearly, we have $\lim _{p \rightarrow 0} \pi(p)=\pi_{m}$ and $\lim _{p \rightarrow \infty} \pi(p)=$ 0 . With $p_{\text {ref }}=p_{1}$, we also have

$\frac{d}{d p} \pi(p)=-a \frac{\exp \left(-b p^{-\eta}\right)}{p^{\eta+1}}$

where $a=\pi_{m} \eta b$ and $b=-p_{1}^{\eta} \ln \left(1-\pi / \pi_{m}\right)$. Because (54) is nonpositive, it follows that as we vary $p$ continuously from 0 to infinity, the values of $\pi(p)$ start off at $\pi_{m}$ and decrease monotonically towards 0 , passing $\pi$ when $p=p_{1}$. Thus, the parameter $\pi_{m}$ is the maximum possible participation fraction, which applies when price is at zero; and, because $\pi_{1}=\pi\left(p_{1}\right)=\pi$, the parameter $\pi$ is the base or initial participation fraction, which applies when price is at $p_{1}$ (note that for any given $\pi_{m}$, there is a one-to-one correspondence between $\kappa$ and $\pi$; see (51)). That $\pi_{m}$ can be less than 1 corresponds to the fact that not necessarily all consumers are ready to make a purchase even if price approaches (or is projected to) 0 . That $\pi(p)$ is decreasing corresponds, of course, to the fact that as price increases, the product becomes less affordable to consumers.

From (54), we immediately have $\lim _{p \rightarrow \infty} d \pi(p) / d p=0$; furthermore, repeated applications of L'Hôpital's rule (details omitted) shows that we also have $\lim _{p \rightarrow 0} d \pi(p) / d p=0$. Thus, (50) is consistent with the natural expectation that price sensitivity should approach 0 when price is either extremely high or very close to 0 .

Upon further differentiation of (54), we have

$\frac{d^{2}}{d p^{2}} \pi(p)=-a \frac{\exp \left(-b p^{-\eta}\right)}{p^{2(\eta+1)}}\left[\eta b-(\eta+1) p^{\eta}\right]$.

Define

$p^{*} \equiv\left(\frac{\eta b}{\eta+1}\right)^{1 / \eta}$

then, it is easily seen that the right-hand side of (55) is nonpositive for $p<p^{*}$, is equal to 0 at $p=p^{*}$, and is nonnegative for $p>p^{*}$. Therefore, the negative of $d \pi(p) / d p$ is unimodal; and this, in turn, implies that as we decrease price, the response of $\pi(p)$ is $\mathrm{S}$-shaped with a unique inflection point at $p=p^{*}$. Thus, (50) is also consistent with the expectation that as price increases, price sensitivity should undergo an initial climb, a levelling phase, and then a final decline.

Next, observe that when $\eta$ approaches $0, \pi(p)$ converges to $\pi$ for all $p$; this means that price sensitivity equals zero in the limit, and we again recover the basic PDM. Hence, (50) is, for any $\pi$, consistent with the basic PDM when 
price sensitivity for a product is low (e.g., when a product has a very low price).

More generally, for any given pair of $\pi_{m}$ and $\pi$, it can be shown (details omitted) that (55) and (56) together imply: (i) if $\eta$ is close to 0 , then, as we increase $p$ from $0, \pi(p)$ starts off horizontally, experiences a sharp drop immediately after the origin, to just above level $\pi$, and then turns nearly flat subsequently; and (ii) if $\eta$ is sufficiently large, then $\pi(p)$ follows a similar pattern, except the sharp downward turn is delayed until $p$ is immediately before $p_{1}$, and it drops all the way past $\pi$ to just above level 0. (Formally, we have $p^{*}$ converges to 0 as $\eta \rightarrow 0$, and to $p_{1}$ as $\eta \rightarrow$ $\infty$.) The significance of (i) and (ii) is that by varying $\eta$, $\pi(p)$ can assume the entire spectrum of slopes in the range $(0,-\infty)$ when prices are in the vicinity of the initial level $p_{1}$. Thus, the parameter $\eta$ serves as the primary control of price sensitivity.

In conclusion, by varying $\pi_{m}, \pi$, and $\eta$ jointly, (50) is capable of accommodating a wide range of price-response patterns. With the incorporation of (50), the PDM now has a total of six parameters, namely, $\pi, \alpha, \beta, \delta, \eta$, and $\pi_{m}$.

4.3.2. Advertising Effects. In general, advertising could have a current-period and a cumulative impact on sales. In this section, we develop formulations that gauge the extent of these impacts.

For current-period impact, we postulate that advertising, like price, modulates the residual market potentials via the $\pi_{i} \mathrm{~s}$. We also assume that a reasonable metric of the extent of the advertising effort in a given time period is the total advertising expenses during that period. For $1 \leqslant$ $i \leqslant n$, let $v_{i}$ be the amount of advertising spending during period $i$; then, the proposed formulation is to scale the term $1-\pi / \pi_{m}$ in (53) (or equivalently, in (50)) to $\left(1-\pi / \pi_{m}\right) \exp \left(-\gamma_{p} v_{i}\right)$, where $\gamma_{p}$, with $\gamma_{p} \geqslant 0$, is a new parameter. This results in

$$
\begin{array}{r}
\pi_{i}=\pi_{m}\left\{1-\exp \left\{-\left[-\ln \left[\left(1-\frac{\pi}{\pi_{m}}\right) \exp \left(-\gamma_{p} v_{i}\right)\right]\right]\right.\right. \\
\left.\left.\cdot\left(\frac{p_{i}}{p_{1}}\right)^{-\eta}\right\}\right\} .
\end{array}
$$

Clearly, for any given $p_{i}$, if $v_{i}=0$, then (57) reduces to (53); that is, without advertising, $\pi_{i}$ is at the base level $\pi\left(p_{i}\right)$ specified by (53). In the presence of advertising, i.e., when $v_{i}$ is positive, (57) says that $\pi_{i}$ is elevated to a value between $\pi\left(p_{i}\right)$ and $\pi_{m}$ (i.e., $\left.\pi(0)\right)$, depending on the magnitude of the parameter $\gamma_{p}$; thus, the extent of the impact of current advertising spending is controlled by $\gamma_{p}$. Moreover, note that the term $1-\pi / \pi_{m}$ (recall that $\pi=\pi\left(p_{1}\right)$ ) in (53) determines via $\kappa$ (see (51) and (52)) the magnitude of $\pi(0)-\pi\left(p_{1}\right)$, i.e., the "width" of the range of possible values for the $\pi_{i}$ s as we vary price. Hence, the multiplier $\exp \left(-\gamma_{p} v_{i}\right)$ in (57) is a width scaling, designed to capture the intuitive belief that greater advertising efforts over time should result in a narrower overall scope of the successive participation fractions. Indeed, if $v_{i} \rightarrow \infty$ for all $i$, then we have $\pi_{i}=\pi_{m}$ for all $i$; that is, price would have little effect on the participation fractions.

A more precise discussion of the rationales behind (57) is now given. Clearly, $\exp \left(-\gamma_{p} v_{i}\right)$ is decreasing in $v_{i}$. It follows that as $v_{i}$ increases from 0 to infinity, while holding other quantities constant, the corresponding values of $\pi_{i}$ in (57) increase monotonically to $\pi_{m}$. Consequently, $\pi_{m}$ continues to serve as the absolute ceiling on the participation fractions. That the convergence of $\pi_{i}$ from $\pi\left(p_{i}\right)$ to $\pi_{m}$ is monotone is, of course, consistent with the common sense that greater advertising effort should result in increased awareness and (hopefully) better reception of a product by consumers. Observe further that (57) can be rewritten as

$\pi_{i}=\pi_{m}\left\{1-\left[1-\frac{\pi\left(p_{i}\right)}{\pi_{m}}\right] \exp \left[-\left(\frac{p_{i}}{p_{1}}\right)^{-\eta} \gamma_{p} v_{i}\right]\right\}$.

Because $\exp \left[-\left(p_{i} / p_{1}\right)^{-\eta} \gamma_{p} v_{i}\right]$ is convex in $v_{i}$, (58) implies that the rate of increase in $\pi_{i}$ is decreasing in $v_{i}$. Hence, (57) also formalizes the rationale that incremental advertising dollars should have diminishing marginal impacts. Finally, a further rearrangement of (58) yields

$$
\frac{\pi_{i}-\pi\left(p_{i}\right)}{\pi_{m}-\pi\left(p_{i}\right)}=1-\exp \left[-\left(\frac{p_{i}}{p_{1}}\right)^{-\eta} \gamma_{p} v_{i}\right] \text {. }
$$

Recall that $\pi_{i}-\pi\left(p_{i}\right)$ is the portion of $\pi_{i}$ that is credited to spending $v_{i}$ on advertising. Because by construction we always have $\pi_{i}-\pi\left(p_{i}\right) \leqslant \pi_{m}-\pi\left(p_{i}\right)$, where $\pi_{m}-\pi\left(p_{i}\right)$ is the maximum possible difference between $\pi_{i}$ and the baselevel $\pi\left(p_{i}\right)$, it follows that the ratio on the left-hand side of (59) can be taken as a proportional measure of the impact of advertising, when price is at $p_{i}$. Furthermore, it is readily seen that for any given amount of advertising spending $v_{i}$, the right-hand side of (59) is decreasing as a function of $p_{i}$. Therefore, (57) explicitly reflects the reasonable expectation that the effectiveness of advertising should decrease as price increases.

To further enhance understanding, a "conceptual" trajectory of the joint price-and-advertising response function (57) is depicted in Figure 3. For simplicity, $v_{i}=v>0$ for all $i$ is assumed. Note that if $v_{i}=v=0$, then we recover the base-level price-response function (53), which corresponds to the lower trajectory in the figure. Moreover, recall from $\S 4.3 .1$ that an important feature of (50) is that it reflects systematic changes in price sensitivity. Thus, these trajectories are S-shaped as price declines. (This is in contrast with Equation (2.14) in Jain and Rao 1990, where $\pi(p)=p^{-\eta}$ is proposed.)

Next, we turn to the formulation of the cumulative impacts of advertising. Recall from $\S 2.1$ that the induction rate $\beta$ corresponds to the total force of influence from an existing adopter, and this force is apportioned across the population. In addition, we have assumed that $\beta$ remains constant over time, independent of the size of the target 
Figure 3. Price-and-advertising response function (Equation (57)).

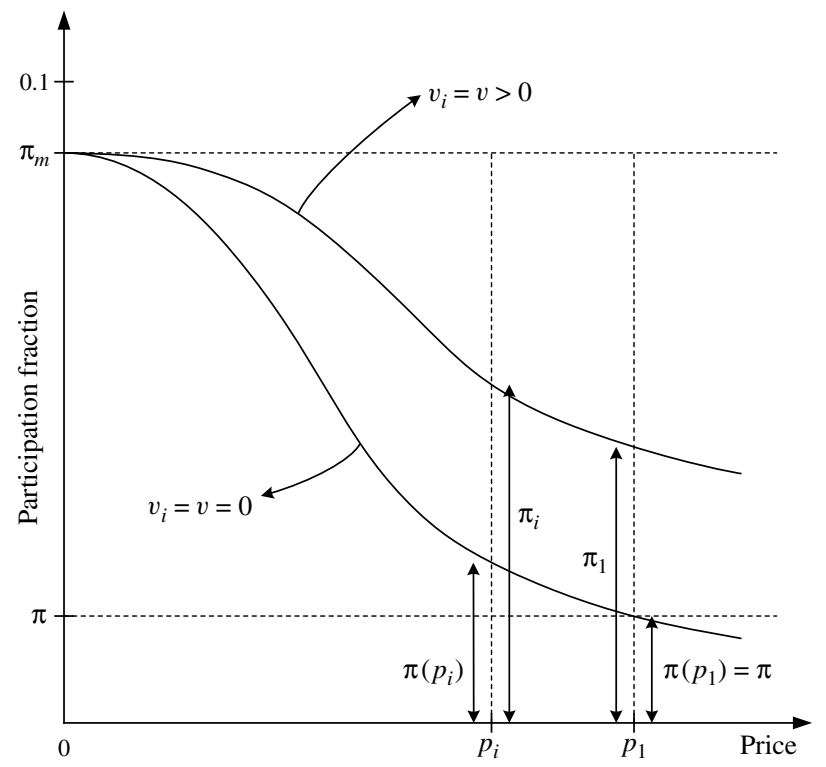

population. In the presence of advertising, it seems plausible that the same level of influence from an existing adopter could result in a greater effect than that in a corresponding scenario without advertising. Furthermore, because potential buyers are subjected to the bombardment of advertising on a continuous basis, it is also plausible that this potential boost in effective influence should depend on the entire advertising history.

Specifically, suppose that the current time period is $i$, $1 \leqslant i \leqslant n$; then, the proposed formulation is to scale $\beta$ in period $i$ to

$\beta\left(1+\gamma_{b} \sum_{j=1}^{i} v_{j}\right)$,

where $\gamma_{b}$, with $\gamma_{b} \geqslant 0$, is a new parameter. In other words, we assume that the boost in effectiveness of the induction force during a time period is proportional to the cumulative sum of all advertising spendings up to and including that period. Note that we continue to assume that the "base" induction rate $\beta$ is constant over all periods.

With the incorporation of both (57) and (60), we now have two more parameters, namely, $\gamma_{p}$ and $\gamma_{b}$, in the PDM.

4.3.3. The Full PDM-Summary. In preparation for empirical analysis, we assemble here a summary of the full PDM with price and advertising. To avoid duplication, we will do so only for the expected-history version; the actualhistory version is similar, and is slightly simpler.

In the expected-history PDM, incremental sales in successive time periods follow the discrete-time stochastic process $\left\{S_{i}, i=1, \ldots, n\right\}$ defined by (41). The mean $\hat{s}_{i}$ and the standard deviation $\hat{\sigma}_{i}$ in (41) are given, respectively, by (44) and (45). In (44) and (45), $t_{i}-t_{i-1}$ is set to $1 ; \pi_{i}$ is specified by (57); $\psi_{i}(t)$, where $t=1$, is given by (18) and (19) with $\alpha$ and $\beta$ replaced by $\hat{\alpha}_{i}$ and $\hat{\beta}_{i} ; \delta_{i}^{2}$ is equal to $\delta^{2}$; and finally, the $\hat{\alpha}_{i} \mathrm{~s}$, the $\hat{\beta}_{i} \mathrm{~s}$, and the $\hat{\mu}_{i}$ s (i.e., the $\hat{s}_{i} \mathrm{~s}$ ) are jointly determined by a revised version of the set of recursions (31)-(33) in which $\pi_{i}$ is specified by (57) and $\beta$ is replaced by (60).

The complete specification of a full PDM is $\left(m, a_{0}\right.$; $\left.\pi, \alpha, \beta, \delta, \eta, \pi_{m}, \gamma_{p}, \gamma_{b}\right)$. Because $m$ and $a_{0}$ are assumed given, we have a total of eight parameters. For specific products, estimates for these parameters are obtained by maximizing the log-likelihood function (47). In addition to $m$ and $a_{0}$, the required inputs are the observed sales $\left\{s_{i}, i=\right.$ $1, \ldots, n\}$, the set of prices $\left\{p_{i}, i=1, \ldots, n\right\}$, and the set of advertising spendings $\left\{v_{i}, i=1, \ldots, n\right\}$.

The full PDM can be specialized easily. To remove advertising effects, we set $\gamma_{p}$ and $\gamma_{b}$ to 0 . To remove price effects, we set $\eta$ to 0 and $\pi_{m}$ to 1 . To recover the basic PDM, we let $\eta=\gamma_{p}=\gamma_{b}=0$ and $\pi_{m}=1$. Finally, to recover the SBM, we let $a_{0}=0$ and $\pi=1$ in the basic $\mathrm{PDM}$, and treat $m$ as a parameter, in addition to $\alpha, \beta$, and $\delta$.

Note that for specific products, not necessarily all of the parameters in the full PDM will test positive. Parameters that are likely to be insignificant include $\alpha, \pi_{m}$, and $\gamma_{b}$; when these cases occur, we will have combinations of $\alpha=0, \pi_{m}=1$, or $\gamma_{b}=0$.

\section{An Empirical Example}

In this section, we report and discuss estimation results for a specific product. As noted in $\S 1$, the BM has been used to study demand growth for a wide variety of products. (Detailed reviews of the existing estimation literature can be found in Mahajan et al. 1986, Bass et al. 2000, and Putsis and Srinivasan 2000.) We will consider the wellstudied example of room air conditioners (RACs) for the period 1949-1961. This example serves as an excellent illustration of the applicability of the PDM.

The PDM (which covers the SBM) is implemented in SAS (SAS Institute; Version 8.02), using its library nonlinear programming (NLP) procedure. Two sample programs are provided in the online companion. For comparison purposes, the BM and the GBM (Bass et al. 1994) are also programmed in SAS. For the BM, the nonlinear-leastsquares (NLS) operationalization (see (48)) in Srinivasan and Mason (1986) is implemented. The GBM is an extension of the BM; it incorporates both price and advertising into the BM via a modification of (3) in which the term $p+q F(t)$ is scaled by a function $x(t)$ that reflects the effect of "current marketing effort." Following the original paper, implementation of the GBM is based on the history-dependent operationalization in Jain and Rao (1990, p. 164, Equation (2.7)). The NLIN procedure in SAS is used to compute the parameter estimates for both the BM and the GBM.

Estimation results for both the actual-history and the expected-history versions of the SBM, the basic PDM 
(PDM-B), the PDM with price (PDM-P; $\gamma_{p}=\gamma_{b}=0$ ), and the full PDM (PDM-F) are summarized in Table 1. The actual sales along with the fitted sales for the three expected-history PDMs are depicted in Figure 4.

For price and advertising, we have taken the data from Table 7 in Bass et al. (1994). From 1949-1961, two sets of sales data have been used in the literature. The results in Table 1 are based on the sales data used in Jain and Rao (1990). This data set is chosen because, for the expectedhistory PDM-F, it yields a better fit than the other data set used in Bass et al. (1994). The corresponding fit statistics for the latter are $R^{2}=0.9938$ and $\mathrm{SSE}=31,616$. The differences in the two data sets are: In the former, sales in 1952, 1955, and 1959 are 365, 1,270, and 1,660 (in thousands), respectively; and in the latter, the corresponding sales are $380,1,267$, and 1,800 (sales in other years are identical). The choice of the data set is, in addition, influenced by the striking observation (see Figure 4) that the expected-history PDM-F is "predicting" lower sales volumes in 1952 and 1959 and a higher sales volume in 1955, and these predictions are, especially in 1959, in lock step with the noted differences between the two sets.

RACs were launched in 1933 (Kohli et al. 1999, Table 1). Prior to 1949, we only have (Electrical Merchandising) scattered sales data that go back to 1936; the available total is about 217. This could be substantially below the actual sales $a_{0}$, which is needed as an input for the PDM. To circumvent this difficulty, a two-step procedure is used to produce the expected-history PDM-F estimates. In the first step, we treat $a_{0}$ as a parameter in the estimation; and this results in an estimate of 744 for $a_{0}$ (with asymptotic standard error 530), which does not seem unreasonable. In the second step, we repeat the estimation with $a_{0}$ fixed at 744; and this yields the final estimates (only asymptotic standard errors are different in the second step; the differences are not substantial) reported in Table 1 . The setting $a_{0}=744$ is then taken as "given" in the estimation of the remaining versions of the PDM.

From Table 1, it can be seen that the two versions of the PDM produce very similar estimates. This is due to the fact that for sufficiently large $m$, the standard deviation of $A_{m}(t)$ is small relative to the mean. For SBM, however, the expected-history version turns out to have a slightlypronounced edge. Conceptually, the actual-history version of a reasonably-specified model is expected to perform better than its expected-history counterpart because there is a greater dependence on actual sales data. This reversal, therefore, suggests that the SBM is not as well specified as the PDMs.

Recall from $\$ 3.2$ that for any given specification, the sales trajectories of the expected-history PDMs are completely independent of the actual sales. In fact, the recursive sequence $\left\{\hat{s}_{i}, i=1, \ldots, n\right\}$ in (44) can be construed as the "closed-form solution" for the expected sales in successive time periods. Consequently, the expected-history versions of the PDM are much more useful in forecasting and planning scenarios where actual sales data are not available. For this reason, we will limit the remainder of the discussion to the expected-history versions.

Table 1 and Figure 4 indicate a very pronounced pattern of progressive improvements of the fitted sales for the PDMs as we add more information. For the expectedhistory PDM-B, we have $R^{2}=0.8861$. That the basic PDM

Table 1. Room air conditioners-SBM and PDM estimates.

\begin{tabular}{|c|c|c|c|c|c|c|c|c|c|c|c|c|}
\hline \multirow[b]{3}{*}{ Model } & \multirow{2}{*}{\multicolumn{2}{|c|}{ Fit statistics }} & \multicolumn{10}{|c|}{ Specification } \\
\hline & & & \multicolumn{2}{|c|}{ Input data } & \multicolumn{8}{|c|}{ Parameters } \\
\hline & $R^{2}$ & $\operatorname{SSE}\left(10^{6}\right)$ & $m\left(10^{3}\right)$ & $a_{0}\left(10^{3}\right)$ & $\pi$ & $\alpha$ & $\beta$ & $\delta$ & $\eta$ & $\pi_{m}$ & $\gamma_{p}$ & $\gamma_{b}$ \\
\hline SBM, AH & 0.9047 & 472,366 & $\begin{array}{l}17,750^{*} \\
{[1,109]}\end{array}$ & - & - & $\begin{array}{c}0.01151 \\
{[0.00526]}\end{array}$ & $\begin{array}{c}0.3932 \\
{[0.0459]}\end{array}$ & $\begin{array}{c}187.57 \\
{[39.41]}\end{array}$ & - & - & - & - \\
\hline SBM, EH & 0.9278 & 357,799 & $\begin{array}{l}18,447^{*} \\
{[1,331]}\end{array}$ & - & - & $\begin{array}{c}0.009626 \\
{[0.002038]}\end{array}$ & $\begin{array}{c}0.3745 \\
{[0.0404]}\end{array}$ & $\begin{array}{l}162.28 \\
{[21.31]}\end{array}$ & - & - & - & - \\
\hline PDM-B, AH & 0.8868 & 560,962 & $53,291^{\dagger}$ & 744 & $\begin{array}{c}0.04297 \\
{[0.00251]}\end{array}$ & n.s. & $\begin{array}{c}10.94 \\
{[1.98]}\end{array}$ & $\begin{array}{l}206.13 \\
{[32.38]}\end{array}$ & - & - & - & - \\
\hline PDM-B, EH & 0.8861 & 564,650 & 53,291 & 744 & $\begin{array}{c}0.04753 \\
{[0.00338]}\end{array}$ & n.s. & $\begin{array}{c}7.942 \\
{[0.990]}\end{array}$ & $\begin{array}{l}206.90 \\
{[39.35]}\end{array}$ & - & - & - & - \\
\hline PDM-P, AH & 0.9700 & 148,934 & 53,291 & 744 & $\begin{array}{c}0.005950 \\
{[0.003248]}\end{array}$ & n.s. & $\begin{array}{c}31.49 \\
{[11.06]}\end{array}$ & $\begin{array}{l}105.93 \\
{[25.36]}\end{array}$ & $\begin{array}{c}9.380 \\
{[2.078]}\end{array}$ & $\begin{array}{c}0.03816 \\
{[0.00054]}\end{array}$ & - & - \\
\hline PDM-P, EH & 0.9720 & 138,854 & 53,291 & 744 & $\begin{array}{c}0.006763 \\
{[0.003328]}\end{array}$ & n.s. & $\begin{array}{l}26.25 \\
{[8.68]}\end{array}$ & $\begin{array}{l}101.93 \\
{[25.32]}\end{array}$ & $\begin{array}{c}9.125 \\
{[1.899]}\end{array}$ & $\begin{array}{c}0.03844 \\
{[0.00064]}\end{array}$ & - & - \\
\hline PDM-F, AH & 0.9959 & 20,507 & 53,291 & 744 & $\begin{array}{c}0.005191 \\
{[0.001208]}\end{array}$ & n.s. & $\begin{array}{l}19.14 \\
{[7.09]}\end{array}$ & $\begin{array}{c}39.52 \\
{[8.83]}\end{array}$ & $\begin{array}{c}6.218 \\
{[1.495]}\end{array}$ & $\begin{array}{c}0.04195 \\
{[0.00487]}\end{array}$ & $\begin{array}{c}0.009746 \\
{[0.001857]}\end{array}$ & $\begin{array}{c}0.3704 \\
{[0.1578]}\end{array}$ \\
\hline PDM-F, EH & 0.9959 & 20,482 & 53,291 & $744^{*}$ & $\begin{array}{c}0.005123 \\
{[0.001229]}\end{array}$ & n.s. & $\begin{array}{l}19.71 \\
{[6.87]}\end{array}$ & $\begin{array}{l}39.56 \\
{[9.00]}\end{array}$ & $\begin{array}{c}6.266 \\
{[1.552]}\end{array}$ & $\begin{array}{c}0.04181 \\
{[0.00479]}\end{array}$ & $\begin{array}{c}0.009733 \\
{[0.001981]}\end{array}$ & $\begin{array}{r}0.3776 \\
{[0.1679]}\end{array}$ \\
\hline
\end{tabular}

Note. AH: Actual-history version, EH: Expected-history version, SSE: Sum of the squared errors, *: A parameter estimate, [...]: Asymptotic standard errors, ${ }^{\dagger}:$ Number of households in 1961, —: Not applicable, n.s.: Not significant. 
Figure 4. Expected-history-PDM fitted sales for RACs.

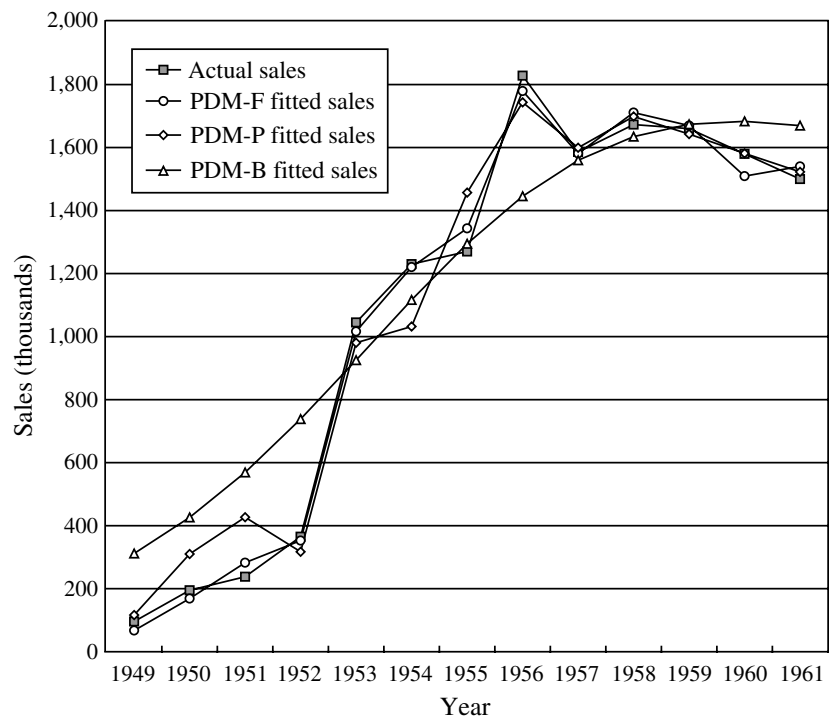

does not produce a very tight fit, especially that the fitted sales from 1949-1952 are several folds higher than the actual sales, is not surprising, as it can be attributed to the fact that prices for the RACs declined $36.8 \%$ (from $\$ 410$ to $\$ 259$, without inflation adjustment) over the course of 13 years. In other words, higher prices during 19491952 should imply lower participation fractions, but this is not reflected in the basic PDM, where the participation fractions are assumed to be constant. Indeed, $R^{2}$ for the expected-history PDM with price is substantially higher, at 0.972. With the further inclusion of advertising, the expected-history PDM-F achieves a remarkable $R^{2}$ of 0.9959 . It is also noteworthy that all of the parameter estimates in the full PDM are quite significant, as indicated by the reported asymptotic standard errors.

For all versions of the PDM, the parameter $\alpha$ turns out to be insignificant. For a newly-launched product, the level of intrinsic interest of consumers certainly is expected to contribute to adoption. However, for products that have existed for a sufficiently long duration prior to $t_{0}$, this contribution may be relatively small when compared to the total influence from the $a_{0}$ existing adopters (i.e., the innovators). Indeed, from (31) (or (26) and (28)), we see that the $\hat{\alpha}_{i} \mathrm{~s}$ are increasing as a function of $i$ (i.e., over time), and that the rate of this increase is proportional to $\beta$. In particular, if $\beta\left(a_{0}+\sum_{j=1}^{i-1} \hat{\mu}_{j}\right) /(m-1)$ is sufficiently large, then it will dominate $\alpha$. For RACs, our first year of study is 1949 , which is 16 years after the launch. That $\alpha$ does not test positive, therefore, is a reflection of the relative contributions of $\alpha$ and $\beta a_{0} /(m-1)$ in (31).

In the expected-history PDM-F, we have $\pi=0.005123$, $\eta=6.266$, and $\pi_{m}=0.04181$. A plot of (50) with these values shows that as price decreases from $p_{1}=410$ to $p_{13}=259$, price sensitivity experiences an initial climb, a slight levelling, and then a slight decline (similar to Figure 3). In other words, we have a reasonably high price sensitivity, as indicated by the value of $\eta$; and this is coupled with a decline in sensitivity towards the tail end of the observed price range. In general, for products with this type of response in a sufficiently-wide price range, the parameter $\pi_{m}$ is expected to play an active role in achieving good accuracy. Indeed, for RACs, $\pi_{m}$ is the most significant parameter in both PDM-P and PDM-F. Note that in the full PDM, the sequence of estimated participation fractions in (57) (with $\gamma_{p}=0.009733$ ), which grows from $\pi_{1}=$ $\pi=0.005123$ to $\pi_{13}=0.038653$, also exhibits a similar S-shaped pattern.

To compare the PDM against previous models, we also provide in Table 2 estimation results for the $\mathrm{BM}$ and the GBM. Fitted sales for the expected-history SBM (also see Table 1), the BM, and the GBM are shown in Figure 5.

It can be seen from Figures 4 and 5 that the fitted sales for the expected-history PDM-F compare very favorably against that for the GBM. Specifically, the respective sums of squared errors in Tables 1 and 2 are 20,482 and 131,496; this corresponds to an $84.4 \%$ marginal reduction. Note that including the variance of the i.i.d. error terms, the GBM has six parameters; in contrast, the full PDM estimates involve seven parameters ( $\alpha$ is not significant). Apart from relying on the same approximation scheme (11) for means, these two models have disparate parameter regimes as well as distinct sampling properties; in particular, the formulations of price and advertising effects are entirely different. Therefore, the remarkable accuracy of the full PDM should not be attributed to the extra parameter count.

Figure 5 also shows that the expected-history SBM and the BM produce nearly identical fitted sales. (The marginal reduction in SSE from SBM/BM to PDM-F is at 94.3\%.) This confirms the quality of approximation (48)

Table 2. Room air conditioners-BM and GBM estimates.

\begin{tabular}{lcccccccc}
\hline & \multicolumn{2}{c}{ Fit statistics } & \multicolumn{5}{c}{ Parameters } \\
\cline { 2 - 5 } \cline { 5 - 7 } Model & $R^{2}$ & SSE $\left(10^{6}\right)$ & & $m\left(10^{3}\right)$ & $p$ & $q$ & $\beta_{1}$ & $\beta_{2}$ \\
\hline BM & 0.9278 & \multirow{2}{*}{357,765} & & 18,469 & 0.00969 & 0.3735 & - & - \\
& & & {$[1,431]$} & {$[0.00214]$} & {$[0.0415]$} & & \\
GBM & 0.9735 & \multirow{2}{*}{131,496} & 19,283 & 0.00514 & 0.3279 & -1.4221 & 0.6531 \\
& & & {$[1,147]$} & {$[0.00150]$} & {$[0.0303]$} & {$[0.6635]$} & {$[0.2657]$} \\
\hline
\end{tabular}

Note. $\beta_{1}$ : price parameter, $\beta_{2}$ : advertising parameter. 
Figure 5. BM, GBM, and expected-history-SBM fitted sales for RACs.

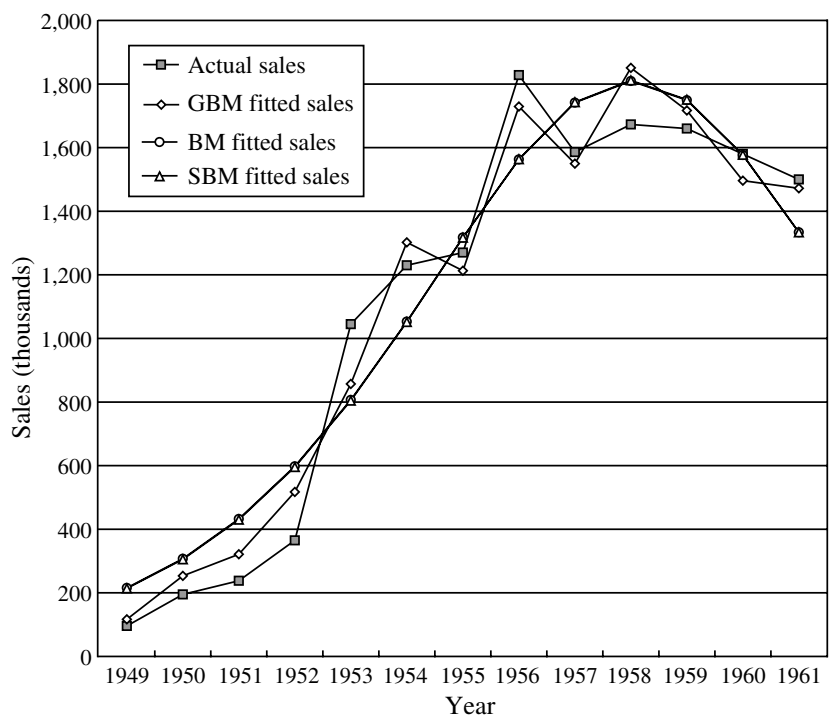

in §4.1. Moreover, the corresponding parameter estimates in Tables 1 and 2 are similarly close. The trajectories of expected sales in these two models can therefore be considered empirically equivalent (when $m$ is large). Note that the SBM is heteroscedastic, and that it yields slightly lower asymptotic standard errors of the estimates.

To assess the predictive ability of the PDM, step-ahead forecasts are also conducted for RACs. The accuracy of forecasts has traditionally been measured by mean absolute deviation (MAD), mean absolute percent deviation (MAPD), and mean squared error (MSE). These forecast statistics for the full PDM, along with those for the GBM, are summarized in Table 3.

In addition to the standard one-step-ahead forecasts, where a single forecast for period $n+1$ is made using the fitted parameters from periods 1 through $n$, Table 3 also reports results for three- and five-step-ahead forecasts. More precisely, this means that joint forecasts for periods $n+j$ with $j$ ranging from 1 to $k$, where $k$ equals 1,3 , or 5 , are made with the estimated parameters "frozen" at period $n$ (for the PDM, the same $m$ is used to conduct all step-ahead forecasts). For $k=1$, the reported statistics are based on iterative forecasts for every specified period, whereas for $k=3$ and $k=5$, on individual components in a single vector of $k$ forecasts conducted without parameter update.

For the PDM, multiperiod forecasts with $k=3$ and $k=5$ are recursively computed using (31)-(33), (57), and (60) over the forecasted periods. As mentioned earlier, regardless of whether the parameters are estimated using the actual-history or the expected-history version, forecasting must be conducted with the latter version, because actual sales data for future periods are not supposed to be available. Note, however, that all forecasts are made with the knowledge of price and advertising information during the forecasted periods. In making "real-life" forecasts, this would have to be projected (or set) separately. The reported results, therefore, provide an indication of how well the PDM performs when we are equipped with the "correct" control information.

From Table 3, it can be seen that the one-step-ahead forecast statistics of the full PDM substantially outperform those of the GBM. For example, the reduction in the MSE, which indicates the extent of variability of the forecasts, is at $96.2 \%$ (7,288 versus 190,349$)$.

In general, forecast statistics with $k=3$ and $k=5$ are expected to be progressively worse than those with $k=1$, as less information is used to produce forecasts over a longer horizon. Results in Table 3 indicate that in terms of MAD, MAPD, and MSE, the PDM-F forecasts are remarkably robust in this regard. Note, in particular, that in terms of both MAD and MAPD, the three-step-ahead forecasts actually turn out to perform better than their one-step-ahead counterparts.

Table 3 also gives the three- and five-step-ahead forecasts for the GBM. Because the Jain and Rao (1990) operationalization of the GBM requires successive cumulative past sales as input, these multiperiod forecasts are made with the actual sales during the forecasted periods. (For one of the forecasted periods, the cumulative past sales of RACs surpasses the estimated $m$, suggesting that the estimate is questionable. The sales forecast for that period is set to 0.) From the reported results, it can be seen that the forecast statistics of the GBM suffer a very rapid deterioration as $k$ increases. This is in marked contrast with the full PDM, which yields MSEs (for example) that are $98.6 \%$ (for $k=3$ ) and $99.3 \%$ (for $k=5$ ) lower.

Finally, a very useful feature of the PDM is that once its parameters are estimated from data, one can easily extract information on the variability of the sales trajectory. Recall

Table 3. Step-ahead forecasts for RACs.

\begin{tabular}{|c|c|c|c|c|c|c|c|}
\hline \multirow{2}{*}{$\begin{array}{l}\text { Forecasted } \\
\text { periods }\end{array}$} & \multirow{2}{*}{$\begin{array}{l}\text { Steps } \\
\text { ahead }\end{array}$} & \multicolumn{3}{|c|}{ PDM-F forecast statistics } & \multicolumn{3}{|c|}{ GBM forecast statistics } \\
\hline & & MAD & MAPD $(\%)$ & MSE & MAD & MAPD $(\%)$ & MSE \\
\hline 1957-1961 & 1 & 64.96 & 4.07 & 7,288 & 386.51 & 24.01 & 190,349 \\
\hline 1959-1961 & 3 & 55.07 & 3.50 & 7,380 & 682.77 & 43.99 & 538,262 \\
\hline 1957-1961 & 5 & 78.89 & 4.94 & 7,855 & 994.11 & 63.04 & $1,163,224$ \\
\hline
\end{tabular}

Note. MAD: Mean absolute deviation, MAPD: Mean absolute percent deviation, MSE: Mean squared error. 
that for period $i$, the variance $\hat{\sigma}_{i}^{2}$ of $S_{i}$, given by (45), is the sum of two components. Denote by $\theta_{i}^{2}$ the first component of this variance; that is, let

$\theta_{i}^{2} \equiv\left[m-\left(a_{0}+\sum_{j=1}^{i-1} \hat{\mu}_{j}\right)\right] \pi_{i} \psi_{i}(1)$

Then, according to (34), (42), and (43), the magnitudes of the $\theta_{i}^{2}$ s convey information regarding the extent of the variability in the trajectory of the $A_{i} \mathrm{~s}$, i.e., of the sales trajectory in the absence of the "current" $D_{i}$ s. To facilitate comparison across different products, we further define $\rho_{i} \equiv \theta_{i}^{2} / \hat{\sigma}_{i}^{2}$, which is then a variability index that expresses the variance of $A_{i}$ as a proportion of the variance of $S_{i}$. The PDM-F fitted variability indices for RACs are depicted in Figure 6. It can be seen that the $\rho_{i}$ s are bell shaped, and that variability of the $A_{i} \mathrm{~s}$ is substantially higher during the active growth periods of the demand for RACs. The ability of the PDM to quantify variability is of particular importance in product-planning and manufacturing settings (see, e.g., Cohen et al. 2000, p. 245).

\section{Conclusion}

The fundamental distinction between the PDM and the BM is that in the former, the observed sales in each period is treated as one particular realization of a diffusion, formalized as a run of an SBM, whereas in the latter, sales for all periods are tied to a single bell-shaped deterministic curve (i.e., $f_{\infty}(t)$ ), under the arguable assumption (see, e.g., Mahajan and Wind 1986b, pp. 15-16, and Mahajan et al. 1990 , p. 11) of full participation of all remaining individuals in the target population, in all periods. An important consequence of this difference in formulation is the shift of emphasis from the estimation of the supposedly generic

Figure 6. PDM-F fitted variability indices for RACs.

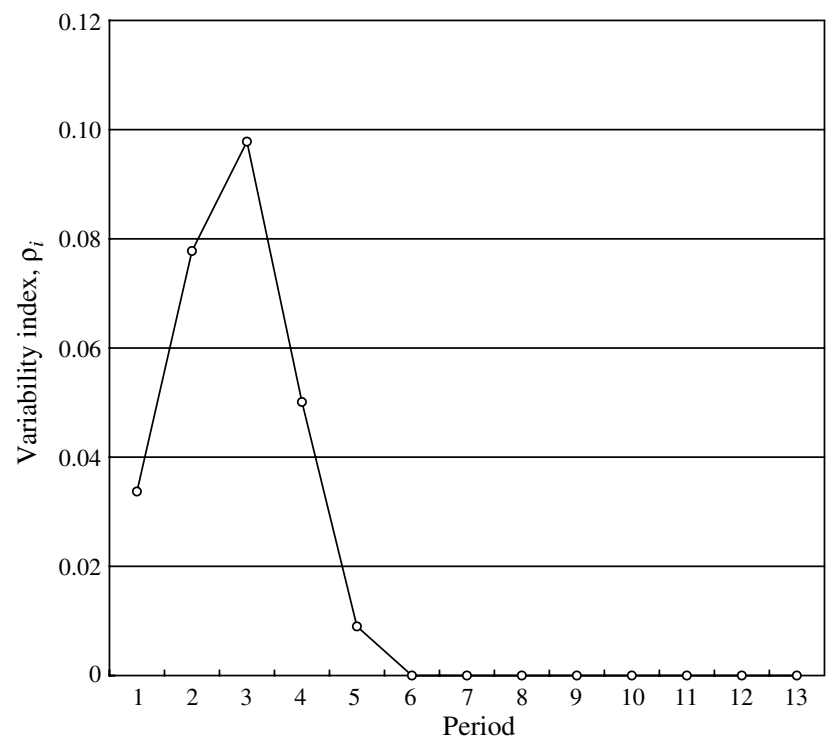

ultimate market potential $m$ to that of the participation fractions over time.

The piecewise and dynamic nature of the PDM makes it highly versatile. This flexibility facilitates, in particular, the incorporation of price and advertising effects into the formulation of the participation fractions. As discussed in $\S \S 4.3 .1$ and 4.3.2, the response function (57) (see Figure 3) has many built-in features that are consistent with commonsense intuition. Indeed, the resulting full PDM is solidly supported by the empirical results in $\$ 5$ (and in Niu 2006). In general, due in part to the lack of relevant data, microlevel stochastic models are seldom validated thoroughly. The PDM-F provides one such example.

With the availability of additional data, other factors (such as supply and/or distribution constraints and quality improvements) that contribute to the readiness of potential adopters can also be incorporated into the formulation of the participation fractions, in manners similar to what has been done for price and advertising.

The micro-level assumptions in the PDM correspond vividly with well-grounded beliefs as to how adopters would behave over time. Consequently, the PDM provides us with the means to better understand the dynamics of a host of underlying demand characteristics. These include the evolution of the induction force from existing adopters (i.e., the $\hat{\alpha}_{i} \mathrm{~s}$ and the $\hat{\beta}_{i} \mathrm{~s}$ ), the price-response curve (i.e., (50)), the incremental current-period and cumulative effects of advertising (i.e., (59) and (60)), the extents of market penetration (i.e., the $m_{i} \mathrm{~s}$, determined by the $\pi_{i} \mathrm{~s}$ ), and the extent of the variability of the sales trajectory (i.e., the $\theta_{i}^{2} \mathrm{~s}$ together with $\delta^{2}$, and the $\rho_{i} \mathrm{~s}$ ). All of these characteristics are readily available once the parameters of the PDM are estimated from data. In addition, the asymptotic standard errors of the parameter estimates allow us to gauge the significance of various underlying forces that contribute to the demand of specific products.

Two useful directions for future research are parallel adoptions of competing brands of similar products (Dolan et al. 1986, Chatterjee et al. 2000) and overlapping life cycles of successive generations of the same functional product (Norton and Bass 1987). The impact of pricing and advertising in such scenarios would naturally be of particular interest. Formulation and solution of vectorvalued versions of the PDM are needed to cope with the resulting interdependent diffusion processes. Another possible direction is to study production and inventory models that integrate the PDM as the demand component (traditional models typically assume that successive demands are independent). These topics will be investigated in subsequent work.

\section{Acknowledgments}

The author is grateful to his colleague Frank M. Bass for extensive help and discussion during the initial stage of this research, and to Hanqin Zhang, of Academic Sinica, 
for numerous discussions related to functional central limit theorems. Thanks are also due to the associate editor and the referees for their efforts in the review of this paper.

\section{References}

Bass, F. M. 1969. A new product growth model for consumer durables. Management Sci. 15 215-227.

Bass, F. M. 1980. The relationship between diffusion rates, experience curves, and demand elasticities for consumer durables technological innovations. J. Bus. 53 51-57.

Bass, F. M., D. C. Jain, T. V. Krishnan. 2000. Modeling the marketing-mix influence in new-product diffusion. V. Mahajan, E. Muller, Y. Wind, eds. New-Product Diffusion Models. Kluwer Academic Publishers, Boston, MA, 99-122.

Bass, F. M., T. V. Krishnan, D. C. Jain. 1994. Why the Bass model fits without decision variables. Marketing Sci. 13 203-223.

Billingsley, P. 1999. Convergence of Probability Measures, 2nd ed. Wiley, New York.

Böker, F. 1987. A stochastic first purchase diffusion model: A counting process approach. J. Marketing Res. 24 64-73.

Chatterjee, R., J. Eliashberg, V. R. Rao. 2000. Dynamic models incorporating competition. V. Mahajan, E. Muller, Y. Wind, eds. NewProduct Diffusion Models. Kluwer Academic Publishers, Boston, MA, 165-205.

Cohen, M. A., T. H. Ho, H. Matsuo. 2000. Operations planning in the presence of innovation-diffusion dynamics. V. Mahajan, E. Muller Y. Wind, eds. New-Product Diffusion Models. Kluwer Academic Publishers, Boston, MA, 237-259.

Dolan, R. J., A. P. Jeuland, E. Muller. 1986. Models of new product diffusion: Extension to competition against existing and potential firms over time. V. Mahajan, Y. Wind, eds. Innovation Diffusion Models of New-Product Acceptance. Ballinger, Cambridge, MA, 117-149.

Eliashberg, J., R. Chatterjee. 1986. Stochastic issues in innovation diffusion models. V. Mahajan, Y. Wind, eds. Innovation Diffusion Models of New-Product Acceptance. Ballinger, Cambridge, MA, 151-203.

Ethier, S. N., T. G. Kurtz. 1986. Markov Processes: Characterization and Convergence. Wiley, New York.

Feller, W. 1971. An Introduction to Probability Theory and Its Applications, Vol. II, 2nd ed. Wiley, New York.

Ho, T.-H., S. Savin, C. Terwiesch. 2002. Managing demand and sales dynamics in new product diffusion under supply constraint. Management Sci. 48 187-206.

Horsky, D., L. Simon. 1983. Advertising and the diffusion of new products. Marketing Sci. 2 1-17.

Jain, D. C., R. C. Rao. 1990. Effect of price on the demand for durables: Modeling, estimation, and findings. J. Bus. Econom. Statist. 8 163-170.

Jeuland, A. P. 1979. Epidemiological modeling of diffusion of innovation: Evaluation and future directions of research. Proc. 1979 Educators Conf., American Marketing Association, Chicago, IL, 274-278.

Kalish, S. 1985. A new product adoption model with price, advertising, and uncertainty. Management Sci. 31 1569-1585.

Kalish, S., S. Sen. 1986. Diffusion models and the marketing mix for single products. V. Mahajan, Y. Wind, eds. Innovation Diffusion Models of New-Product Acceptance. Ballinger, Cambridge, MA, 87-115.

Kohli, R., D. R. Lehmann, J. Pae. 1999. Extent and impact of incubation time in new product diffusion. J. Product Innovation Management 16 134-144.
Krishnan, T. V., F. M. Bass, D. C. Jain. 1999. Optimal pricing strategy for new products. Management Sci. 45 1650-1663.

Kumar, S., J. M. Swaminathan. 2003. Diffusion of innovations under supply constraints. Oper. Res. 51 866-879.

Kurawarwala, A. A., H. Matsuo. 1996. Forecasting and inventory management of short life-cycle products. Oper. Res. 44 131-150.

Mahajan, V., E. Muller. 1979. Innovation diffusion and new-product growth models in marketing. J. Marketing 43 55-68.

Mahajan, V., R. A. Peterson. 1985. Models for Innovation Diffusion. Sage, Beverly Hills, CA.

Mahajan, V., Y. Wind. 1986a. Innovation Diffusion Models of NewProduct Acceptance. Ballinger, Cambridge, MA.

Mahajan, V., Y. Wind. 1986b. Innovation diffusion models of new product acceptance: A reexamination. V. Mahajan, Y. Wind, eds. Innovation Diffusion Models of New-Product Acceptance. Ballinger, Cambridge, MA, 3-25.

Mahajan, V., C. H. Mason, V. Srinivasan. 1986. An evaluation of estimation procedures for new product diffusion models. V. Mahajan, Y. Wind, eds. Innovation Diffusion Models of New-Product Acceptance. Ballinger, Cambridge, MA, 203-232.

Mahajan, V., E. Muller, F. M. Bass. 1990. New product diffusion models in marketing: A review and directions for research. J. Marketing $\mathbf{5 4}$ $1-26$.

Mahajan, V., E. Muller, Y. Wind. 2000. New-Product Diffusion Models. Kluwer Academic Publishers, Boston, MA.

Niu, S.-C. 2002. A stochastic formulation of the Bass model of newproduct diffusion. Math. Problems in Engrg. 8 249-263. (Extended abstract and working paper in Rev. Marketing Sci. 1(4) 2002.)

Niu, S.-C. 2006. A piecewise-diffusion model of new-product demands: An empirical study. Paper in preparation.

Norton, J. V., F. M. Bass. 1987. A diffusion theory model of adoption and substitution for successive generations of high-technology products. Management Sci. 33 1069-1086.

Parker, P. M. 1994. Aggregate diffusion forecasting models in marketing: A critical review. Internat. J. Forecasting 10 353-380.

Putsis, W. P., Jr., V. Srinivasan. 2000. Estimation techniques for macro diffusion models. V. Mahajan, E. Muller, Y. Wind, eds. NewProduct Diffusion Models. Kluwer Academic Publishers, Boston, MA, 263-291.

Robinson, B., C. Lakhani. 1975. Dynamic price models for new product planning. Management Sci. 21 1113-1122.

Rogers, E. M. 1995. Diffusion of Innovations, 4th ed. Free Press, New York

Ross, S. M. 2003. Introduction to Probability Models, 8th ed. Academic Press, New York.

Schmittlein, D. C., V. Mahajan. 1982. Maximum likelihood estimation for an innovation diffusion model of new-product acceptance. Marketing Sci. 1 57-78.

Skiadas, C. H., A. N. Giovanis. 1997. A stochastic Bass innovation diffusion model for studying the growth of electricity consumption in Greece. Appl. Stochastic Models Data Anal. 13 85-101.

Srinivasan, V., C. H. Mason. 1986. Nonlinear least squares estimation of new-product diffusion models. Marketing Sci. 5 169-178.

Taga, Y., K. Isii. 1959. On a stochastic model concerning the pattern of communication-Diffusion of news in a social group. Ann. Inst. Statist. Math. 11 25-43.

Trajtenberg, M., S. Yitzhaki. 1989. The diffusion of innovation: A methodological reappraisal. J. Bus. Econom. Statist. 7 35-47. 